The Astronomical Journal, 130:2392-2414, 2005 November

(C) 2005. The American Astronomical Society. All rights reserved. Printed in U.S.A.

\title{
NEPTUNE'S MIGRATION INTO A STIRRED-UP KUIPER BELT: A DETAILED COMPARISON OF SIMULATIONS TO OBSERVATIONS
}

\author{
JOSEPH M. HAHN ${ }^{1}$ \\ Department of Astronomy and Physics, Institute for Computational Astrophysics, Saint Mary's University, \\ Halifax, NS B3H 3C3, Canada; jhahn@ap.stmarys.ca \\ AND \\ Renu MaLhotra \\ Department of Planetary Sciences, University of Arizona, 1629 East University Boulevard, \\ Tucson, AZ 85721-0092; renu@1pl.arizona.edu \\ Received 2004 December 2; accepted 2005 July 12
}

\begin{abstract}
We use $N$-body simulations to examine the consequences of Neptune's outward migration into the Kuiper Belt, with the simulated end states being compared rigorously and quantitatively to the observations. These simulations confirm the 2003 findings of Chiang and coworkers, who showed that Neptune's migration into a previously stirredup Kuiper Belt can account for the Kuiper Belt objects (KBOs) known to librate at Neptune's 5:2 resonance. We also find that capture is possible at many other weak, high-order mean-motion resonances, such as 11:6, 13:7, 13:6, $9: 4,7: 3,12: 5,8: 3,3: 1,7: 2$, and $4: 1$. The more distant of these resonances, such as the $9: 4,7: 3,5: 2$, and $3: 1$, can also capture particles in stable, eccentric orbits beyond $50 \mathrm{AU}$, in the region of phase space conventionally known as the "Scattered Disk." Indeed, $90 \%$ of the simulated particles that persist over the age of the solar system in the Scattered-Disk zone never had a close encounter with Neptune but instead were promoted into these eccentric orbits by Neptune's resonances during the migration epoch. This indicates that the observed Scattered Disk might not be so scattered. This model also produced only a handful of Centaurs, all of which originated at Neptune's meanmotion resonances in the Kuiper Belt. However, a noteworthy deficiency of the migration model considered here is that it does not account for the observed abundance of Main Belt KBOs having inclinations higher than $15^{\circ}$. In order to rigorously compare the model end state with the observed Kuiper Belt in a manner that accounts for telescopic selection effects, Monte Carlo methods are used to assign sizes and magnitudes to the simulated particles that survive over the age of the solar system. If the model considered here is indeed representative of the outer solar system's early history, then the following conclusions are obtained: (1) The observed 3:2 and 2:1 resonant populations are both depleted by a factor of $\sim 20$ relative to model expectations; this depletion is likely due to unmodeled effects, possibly perturbations by other large planetesimals. (2) The size distribution of those KBOs inhabiting the $3: 2$ resonance is significantly shallower than the Main Belt's size distribution. (3) The total number of KBOs having radii $R>50 \mathrm{~km}$ and orbiting interior to Neptune's $2: 1$ resonance is $N \sim 1.7 \times 10^{5}$; these bodies have a total mass of $M \sim 0.08\left(\rho / 1 \mathrm{~g} \mathrm{~cm}^{-3}\right)(p / 0.04)^{-3 / 2} M_{\oplus}$, assuming they have a material density $\rho$ and an albedo $p$. We also report estimates of the abundances and masses of the Belt's various subpopulations (e.g., the resonant KBOs, the Main Belt, and the so-called Scattered Disk) and provide upper limits on the abundance of Centaurs and Neptune's Trojans, as well as upper limits on the sizes and abundances of hypothetical KBOs that might inhabit the $a>50$ AU zone.
\end{abstract}

Key words: Kuiper Belt — methods: $n$-body simulations — solar system: formation

\section{INTRODUCTION}

The Kuiper Belt is the vast swarm of small bodies that inhabit the outer solar system beyond the orbit of Neptune. The Kuiper Belt objects (KBOs) are relics of the solar system's primordial planetesimal disk; they are bits of debris that failed to coalesce into other large planets. The Kuiper Belt is also of great interest because it preserves a record of the outer solar system's early dynamical history. This is reflected in the KBOs' curious distribution of orbits, which suggest that there was considerable readjustment of the solar system's early architecture. The possibility that the orbits of the giant planets may have shifted significantly (that is, after the solar nebula gas had already dissipated) was first demonstrated by the accretion simulations of Fernández \& Ip (1984); they showed that as the growing giant planets gravitationally scatter the residual planetesimal debris,

\footnotetext{
${ }^{1}$ Canada Research Chair in Astrophysics.
}

they can exchange angular momentum with the debris disk in a way that causes the planets' orbits to drift. Malhotra (1993b) later showed that an episode of outward migration by Neptune by at least $\Delta a \simeq 5 \mathrm{AU}$ could also account for Pluto's peculiar orbit, which resides at Neptune's 3:2 resonance with an eccentricity of $e \simeq 0.25$. In this scenario, Pluto's large eccentricity is a consequence of it having been captured by Neptune's advancing $3: 2$ resonance, which pumped Pluto's $e$ up as it shepherded the small planet outward. Further support for this planet-migration scenario is provided by the subsequent discovery of numerous other KBOs also inhabiting Neptune's 3:2 resonance with eccentricities similar to model predictions (Malhotra 1995), as well as by more modern $N$-body simulations of the orbital evolution of giant planets while they are still embedded in a massive planetesimal disk (Hahn \& Malhotra 1999; Gomes et al. 2004).

The purpose of the present work is to use higher resolution simulations to update this conventional model of Neptune's 
migration into the Kuiper Belt. This model's strengths, as well as its weaknesses, are assessed quantitatively by rigorously comparing the simulations' end states to current observations of the Belt. In the following, we execute two simulations that track the orbital evolution of the four migrating giant planets plus $10^{4}$ massless test particles (the latter representing the KBOs) over the age of the solar system. In one simulation the initial state of the Kuiper Belt is dynamically cold (i.e., the particles have initial eccentricities and inclinations of $e$ and $\sin i \sim 0.001$ ), while the second simulation is of a Kuiper Belt that is initially stirred up a modest amount (i.e., $e$ and $\sin i \sim 0.1$ ). We then use a Monte Carlo method to assign sizes (and hence magnitudes) to the simulated KBOs; this allows us to account for the telescopic biases that tend to select those KBOs that inhabit orbits that are more favorable for discovery over those KBOs in less favorable orbits. Then, by comparing the resulting model Kuiper Belts with the current observational data, we rigorously test the planet-migration scenario, as well as obtain a more realistic assessment of the abundance of KBOs. This analysis also provides the relative abundance of the Belt's various subpopulations: the resonant KBOs, the Main Belt objects, and the Scattered Disk, plus the Centaurs and Neptune's Trojans.

The paper is organized as follows: Section 2 describes the so-called standard model considered here, as well as the numerical methods to be employed. Results from the two simulations of the Kuiper Belt are reported in $\S \S 3$ and 4 , while $\S 5$ examines the Kuiper Belt inclination problem. Section 6 details the Monte Carlo model that is then used in $\S \S 7-10$ to assess the relative abundance of the Belt's various subpopulations, with a final tally of the Belt's total population given in $\S 11$. Section 12 comments on some important unmodeled effects, and $\S 13$ summarizes the results.

\section{SIMULATING PLANET MIGRATION}

The MERCURY6 $N$-body integrator (Chambers 1999) is used to track the orbital evolution of the four giant planets plus numerous massless particles. In our simulations, planet migration is implemented by applying an external torque to each planet's orbit so that its semimajor axis $a_{j}$ varies as

$$
a_{j}(t)=a_{f, j}-\Delta_{j} e^{-t / \tau}
$$

where $a_{f, j}$ is planet $j$ 's final semimajor axis, $\Delta_{j}$ is the planet's net radial displacement, and $\tau$ is the $e$-fold timescale for planet migration; this form of planet migration was first used in Malhotra (1993b). To implement this in MERCURY6, the integrator is modified so that each planet's velocity $v_{j}$ is incremented by the small velocity kick

$$
\Delta v_{j}=\frac{1}{2} \frac{\Delta_{j}}{a_{j}} \frac{\Delta t}{\tau} e^{-t / \tau} v_{j}
$$

with each time step $\Delta t$. This additional velocity kick is directed along the planet's velocity vector and results in a torque $T_{j}=$ $m_{j} a_{j} \Delta v_{j} / \Delta t$ being applied to the planet. Since $T_{j}=d L_{j} / d t$, where $L_{j}$ is the planet's angular momentum, these velocity kicks cause the planet's orbit to vary at the rate $\dot{a}_{j}=2 a_{j} T_{j} / L_{j}=\left(\Delta_{j} / \tau\right) e^{-t / \tau}$, which then recovers equation (1) when integrated.

The simulations reported below adopt the current planets' masses and orbits as initial conditions, except that their initial semimajor axes are displaced by an amount $-\Delta_{j}$ so that the migration torque ultimately delivers these planets into orbits similar to their present ones. The free parameters that describe this migration are the planets' radial displacements $\Delta_{j}$ and the migration timescale $\tau$. At present, there is only one strong constraint on the $\Delta_{j}$ values, namely, that Neptune's orbit must expand by $\Delta_{\mathrm{N}} \simeq 8.7 \mathrm{AU}$ if resonance trapping is to account for the KBOs having eccentricities of $0<e \lesssim 0.32$ at Neptune's $3: 2$ resonance (see Appendix A). Another constraint, on the magnitude of Jupiter's inward migration, can be obtained from the orbital distribution of asteroids in the outer asteroid belt. Liou \& Malhotra (1997) show that the severe depletion of the outer asteroid belt can be explained if Jupiter migrated inward by at least $0.2 \mathrm{AU}$, and Franklin et al. (2004) show that the orbits of the Hilda asteroids at Jupiter's 3:2 resonance are consistent with Jupiter having migrated inward by about 0.45 AU. The remaining $\Delta_{j}$ values for Saturn and Uranus are less well constrained, but stability considerations require them to be neither too large nor too small. With this in mind, our simulations adopt the following values for the $\Delta_{j}$ values: $\Delta_{\mathrm{J}}=0.900$ AU for Jupiter, $\Delta_{\mathrm{S}}=2.09 \mathrm{AU}$ for Saturn, $\Delta_{\mathrm{U}}=4.52 \mathrm{AU}$ for Uranus, and $\Delta_{\mathrm{N}}=$ 8.70 AU for Neptune. All the simulations reported here also employ a planet-migration timescale of $\tau=10^{7} \mathrm{yr}$. This value is supported by the self-consistent $N$-body simulations by Hahn \& Malhotra (1999) of the giant planets' migration while they are embedded in a planetesimal disk. Those simulations show that a planetesimal disk having a mass $M_{D} \sim 50 M_{\oplus}$ spread over $10 \mathrm{AU}<a<50 \mathrm{AU}$ will cause Neptune's orbit to expand by $\Delta a \sim 7$ AU over a characteristic timescale of $\tau \sim 10^{7} \mathrm{yr}$ (see also Gomes et al. 2004).

We note that the orbital evolution adopted here is constructed so that the migrating planets' eccentricities are always comparable to their present values and that the migration proceeds along nearly circular orbits. But this particular choice for the planets' eccentricities is merely a simplifying assumption, since we do not know the $e$-evolution of the giant planets during the migration epoch. For instance, it is possible that dynamical friction with the particle disk would have conspired to keep the planets' eccentricities low, but there may also have been other transient protoplanets roaming about the outer solar system, and their perturbations would tend to pump up the planets' eccentricities. Given the uncertainty in the relative rates of these effects, we adopt the simplest possible model, one that assumes that the giant planets' eccentricities were always comparable to their present ones. However, alternate migration schemes are possible; for instance, Tsiganis et al. (2005) consider a scenario in which the giant planets pump up their eccentricities as they pass through mutual resonances. But it is uncertain as to whether this possible history would have altered the bulk properties of the Kuiper Belt, and it is not considered here.

In order to enforce migration in nearly circular orbits, our simulations have Jupiter migrating outward $\Delta_{\mathrm{J}}=0.9 \mathrm{AU}$, whereas other self-consistent simulations show that Jupiter usually migrates inward a small amount (Hahn \& Malhotra 1999). Note that this choice avoids having Jupiter approach the 5:2 resonance with Saturn, which tends to excite the planets' eccentricities above current levels. However, that eccentricity excitation might then have been damped back to current levels by dynamical friction with the particle disk, but that is a phenomenon that goes unmodeled in our massless particle disk. We simply avoid this event altogether by instead having Jupiter migrate outward a modest amount. But this not a concern here, since our interest is in the Kuiper Belt, whose end state is not likely to preserve any memory of whether Jupiter migrated slightly inward or outward. The remaining $\Delta_{j}$ values are similarly chosen to avoid all major resonances, and the simulated planets' semimajor axes $a_{j}(t)$ are also shown in Figure 1. 


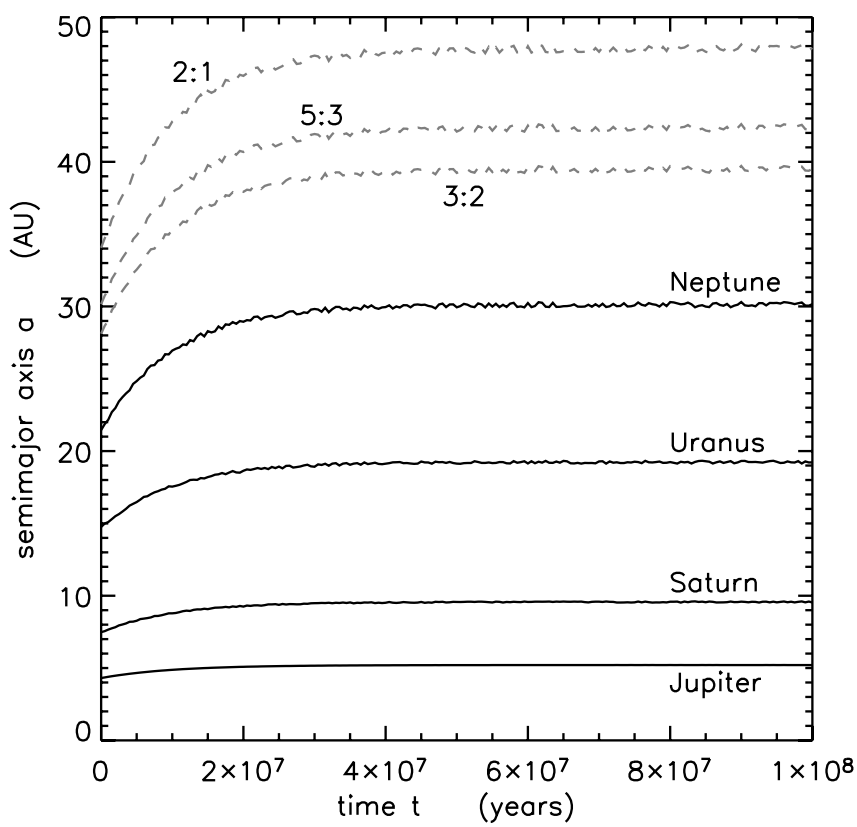

FIG. 1.-Planets' semimajor axes $a_{j}$ vs. time $t$, as well as a few of Neptune's mean-motion resonances that are effective at trapping particles.

In the simulations described below, the model Kuiper Belt is initially composed of $10^{4}$ massless particles having semimajor axes randomly distributed over $20 \mathrm{AU}<a<80$ AU, with a surface number density that varies as $a^{-2}$. The inner edge of this particle disk is 1.4 AU inward of Neptune's initial semimajor axis, and the disk extends well beyond the outer reaches of the observed Main Belt. All simulations described here use a time step of $\Delta t=0.5 \mathrm{yr}$, which is sufficiently short to accurately evolve particles in eccentric orbits down to perihelia as low as $q_{\min } \simeq(10 \Delta t / 1 \mathrm{yr})^{2 / 3} \mathrm{AU} \simeq 3 \mathrm{AU}$ without suffering the perihelion instability ${ }^{2}$ described in Rauch \& Holman (1999). Of course, particles can still achieve orbits having perihelia lower than $q_{\text {min }}$, but their orbits will not be calculated correctly in our simulations. However, this is of little consequence, since these planet-crossing bodies have very short dynamical lifetimes and are quickly removed from the system anyway.

\section{MIGRATION INTO A DYNAMICALLY COLD KUIPER BELT}

Accretion models have shown that the observed KBO population must have formed in an environment that was initially dynamically cold, that is, the known KBOs must have formed from seeds that were in nearly circular and coplanar orbits with initial $e$ and $\sin i$ values that were $\lesssim 10^{-3}$ (Stern 1996; Kenyon \& Luu 1999). In anticipation of this, many investigations of the dynamical history of the Kuiper Belt have adopted initial KBO orbits that are dynamically cold (e.g., Malhotra 1993b, 1995; Duncan et al. 1995; Yu \& Tremaine 1999; Chiang \& Jordan 2002).

Figure 2 shows the results of a simulation of Neptune's smooth migration into a dynamically cold swarm of massless KBOs having initial $e$-values that are Rayleigh-distributed about a mean value $\langle e\rangle=0.001$ and initial inclinations similarly distributed with a mean $\langle\sin i\rangle=\langle e\rangle / 2$. This system is evolved for $t=5 \times 10^{8} \mathrm{yr}$. As is well known from previous studies,

\footnotetext{
2 This difficulty is overcome by the algorithm of Levison \& Duncan (2000).
}

Neptune's smooth migration is very efficient at inserting particles into the planet's mean-motion resonances, principally the $2: 1,5: 3$, and $3: 2$ resonances. However, it is also well recognized that the end state of this idealized model differs from the observed KBO orbits (Fig. 2, red circles) in several ways. For example, one prominent discrepancy is that the $2: 1$ resonance is densely populated with simulated particles, while only sparsely populated by observed KBOs.

However, the discrepancy that is most important to this discussion lies in the $44 \mathrm{AU}<a<47 \mathrm{AU}$ zone between the $7: 4$ and the $2: 1$ resonances, which is the outer half of the Main Belt, conventionally defined as the region between the $3: 2$ and 2:1 resonances. Although these simulated particles managed to slip through the advancing 2:1 resonance, they still reside in orbits that are only modestly disturbed, with $e \sim 0.05$ and $i \sim 0.5$, whereas the observed KBOs inhabit orbits that are considerably more excited. Thus, Neptune's smooth migration into a dynamically cold Kuiper Belt is unable to account for the Belt's stirred-up state.

Evidently, some other event has also disturbed the Kuiper Belt, and this stirring event may have taken place prior to or after the onset of Neptune's migration. However, $\S 4$ provides reason to believe that this stirring event occurred before the onset of Neptune's migration into the Kuiper Belt.

\section{MIGRATION INTO A STIRRED-UP BELT}

To examine the effects of Neptune's migration and its resonance sweeping of a previously stirred-up Kuiper Belt population, we repeat the numerical integrations with $10^{4}$ simulated KBOs but with initial $e$-values Rayleigh-distributed about a mean value of $\langle e\rangle=0.1$ and initial $i$-values distributed similarly about a mean value of $\langle\sin i\rangle=\langle e\rangle / 2$. However, this time the simulation is evolved for the age of the solar system, $4.5 \mathrm{Gyr}$, with Figure 3 showing the resulting Kuiper Belt end state.

First we note that in this case we find an outer solar system that is far more depleted in transient particles such as Centaurs (which are scattered particles having semimajor axes interior to Neptune) and Scattered Disk objects (which are particles that were lofted into eccentric Neptune-crossing orbits due to a close encounter with Neptune [Duncan \& Levison 1997]); those bodies usually reside in orbits having perihelia $q$ between the $q=$ 30 and $q=40$ AU curves seen in Figures 2 and 3. This difference is primarily due to the simulation's longer integration time.

Another prominent difference with the "cold belt" simulation is that Neptune's weaker higher order resonances, such as $3: 1$ and $5: 2$, are considerably more efficient at capturing particles when Neptune migrates into a hot disk, a phenomenon that was first noted by Chiang et al. (2003). This result was rather surprising, because low-order resonance capture theory predicts a generally lower capture probability for particles having higher eccentricities (Borderies \& Goldreich 1984; Malhotra 1993a). However, a careful examination of the theory of adiabatic resonance capture (e.g., Dermott et al. 1988) shows that there are two reasons for this result: (1) The higher order resonances have capture probabilities that drop off more slowly with eccentricity than first-order resonances; although the first-order resonance capture probability varies as $\sim e^{-3 / 2}$, the second-order resonance capture probability falls off more slowly, as $\sim e^{-1}$, while the third-order resonance capture probability varies as $\sim e^{-1 / 2}$. (2) The threshold migration speeds for adiabatic resonance capture are also lower for the higher order resonances, and they also depend more strongly on the initial eccentricities. For capture at a $j+k: j$ resonance, the requirement for adiabatic resonance capture is that Neptune's 

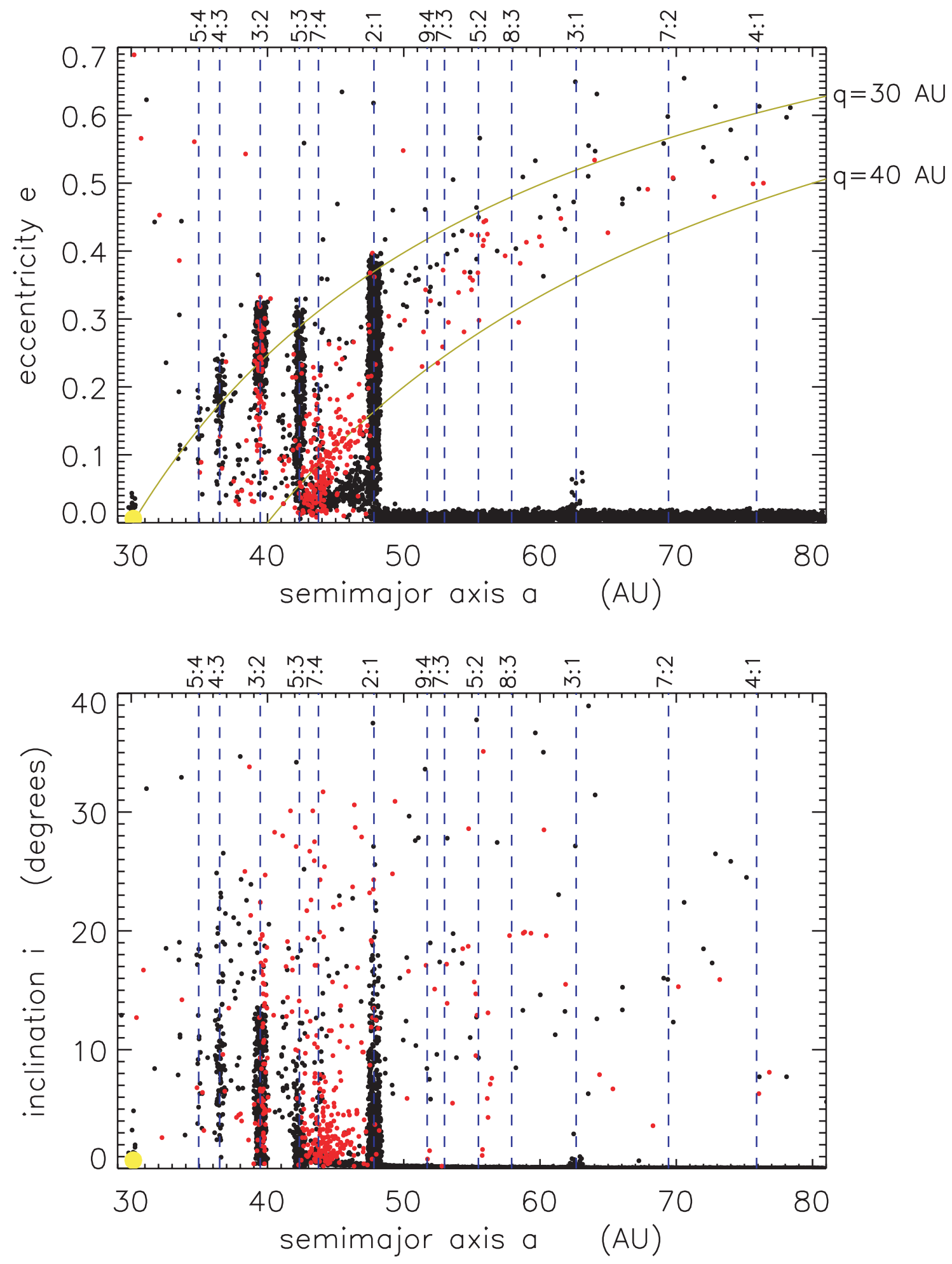

FIG. 2.-Simulation of Neptune's migration into a dynamically cold Kuiper Belt. The model Kuiper Belt is initially composed of $10^{4}$ massless particles with semimajor axes randomly distributed over $20 \mathrm{AU}<a<80 \mathrm{AU}$ and a surface number density that varies as $a^{-2}$. The particles' initial eccentricities are Rayleighdistributed about a mean $\langle e\rangle=0.001$, and their initial inclinations (which are measured with respect to the system's invariable plane) are similarly distributed over $\langle\sin i\rangle=\langle e\rangle / 2$, while their other angular orbital elements are uniformly distributed over $2 \pi$. The four giant planets' orbits migrate according to the prescription described in $\S 2$, and the black circles show the simulated Kuiper Belt end state: the particles' $e$-and $i$-values vs. $a$ at time $t=5 \times 10^{8}$ yr. The red circles show the ecliptic orbital elements of KBOs reported by the Minor Planet Center as having been observed for at least two oppositions. The yellow circles indicate Neptune's orbit, and the vertical dashed lines show the locations of Neptune's various $j+k: j$ mean-motion resonances. Orbits having perihelia $q=30$ and 40 AU are also indicated by the curves. 

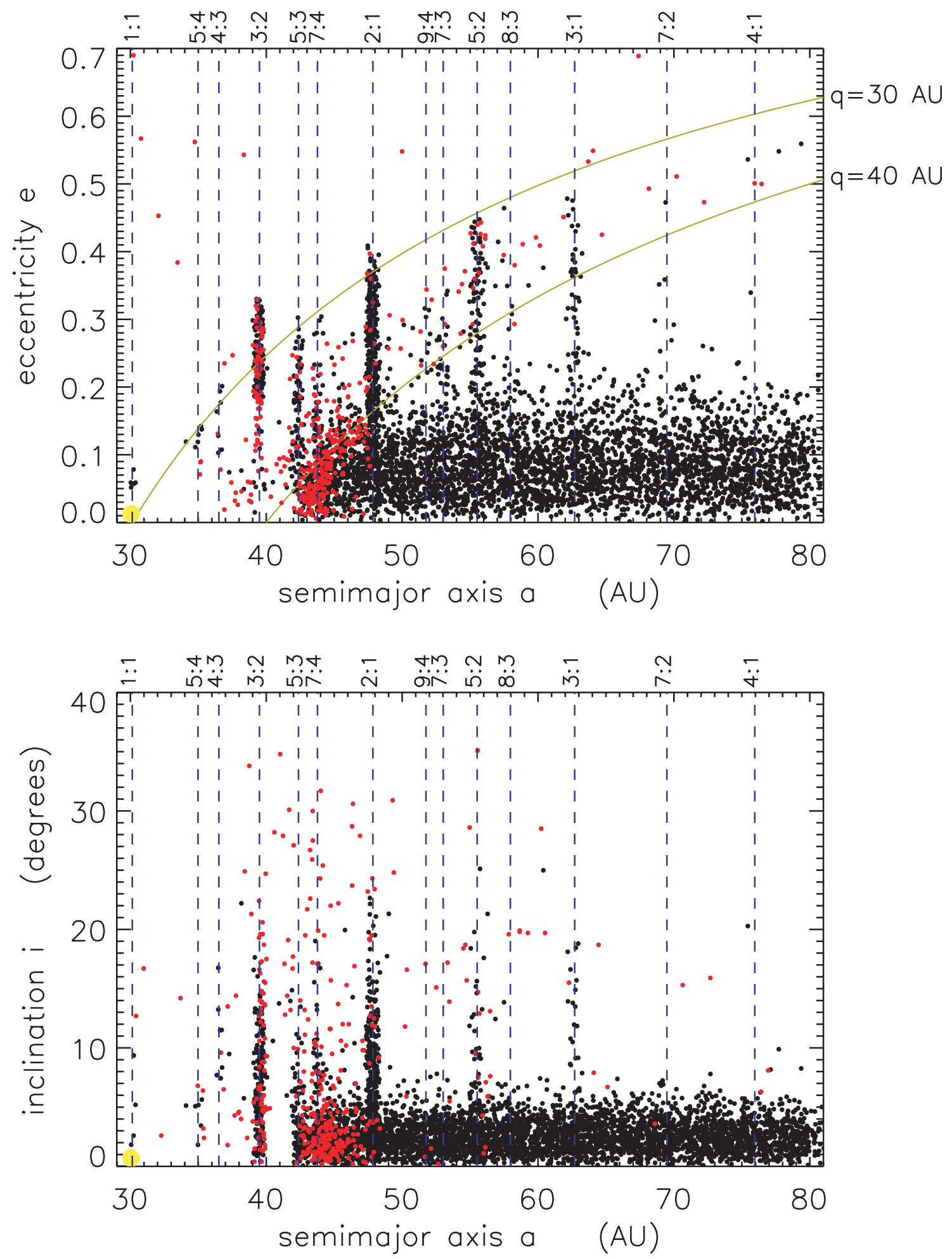

FIG. 3.-Neptune migration into a dynamically hot disk of $N=10^{4}$ particles with initial $e$-values Rayleigh-distributed about $\langle e\rangle=0.1$ and inclinations similarly distributed about $\langle\sin i\rangle=\langle e\rangle / 2$, while all other initial orbits are distributed as in Fig. 2. This system is evolved for $t=4.5 \times 10^{9} \mathrm{yr}$. 
migration rate, which is $\dot{a}_{\mathrm{N}} \sim \Delta_{\mathrm{N}} / \tau \sim 10^{-6} \mathrm{AU} \mathrm{yr}^{-1}$ in these simulations, be sufficiently slow, namely, that

$$
\left|\dot{a}_{\mathrm{N}}\right| \ll 8 j C_{j k} e^{k} \frac{m_{\mathrm{N}}}{M_{\odot}} \frac{a_{\mathrm{N}}}{P_{\mathrm{N}}},
$$

where $m_{\mathrm{N}}$ and $P_{\mathrm{N}}$ are Neptune's mass and orbital period and $C_{j k}$ is a function of Laplace coefficients. For example, the 5:2 resonance has $C_{23} \simeq 3.3$, so the migration speed threshold that permits adiabatic resonance sweeping is $\left|\dot{a}_{\mathrm{N}}\right| \ll 5 \times 10^{-7} \mathrm{AU} \mathrm{yr}^{-1}$ among particles having $e \sim 0.1$, while the threshold is reduced to $\left|\dot{a}_{\mathrm{N}}\right| \ll 5 \times 10^{-13} \mathrm{AU} \mathrm{yr}^{-1}$ among particles having $e \sim 0.001$. It is clear then that a dynamically cold particle swarm has no chance of adiabatic capture at Neptune's high-order meanmotion resonances, while particles that are stirred up to $e \sim 0.1$ are at least near the threshold for adiabatic resonance capture. And as Chiang et al. (2003) point out, the fact that seven eccentric KBOs are known to librate at Neptune's 5:2 resonance also lends support to the prestirred Kuiper Belt scenario.

Another advantage of this stirred-up Kuiper Belt scenario is that it recovers eccentricities that are observed to be as large as $e \sim 0.2$ in the Main Belt, which lies between the 3:2 and 2:1 resonances at $40 \mathrm{AU} \lesssim a \lesssim 48 \mathrm{AU}$ (Fig. 3, red circles). This is a feature that the cold Belt scenario (Fig. 2) does not account for. Of course, Figure 3 also shows that the simulated Main Belt is densely populated by low-eccentricity particles having $e \sim$ 0.05 at $a \sim 47 \mathrm{AU}$, whereas the observed Kuiper Belt is only sparsely populated here. But $\S 7$ shows that there are a variety of possible explanations for this discrepancy - such as a change in the KBO size distribution or perhaps an outer edge in the primordial Kuiper Belt.

Figure 3 also shows that trapping at the distant high-order resonances such as 5:2 and 3:1 is quite effective at promoting bodies into eccentric orbits having $a \gtrsim 50$ AU and perihelia $30 \mathrm{AU} \lesssim q \lesssim 40 \mathrm{AU}$. This domain is usually regarded as the Scattered Disk. This result then suggests the possibility that some of the observed KBOs in the $30 \mathrm{AU} \lesssim q \lesssim 40$ AU zone may actually be resonantly trapped bodies that are masquerading as members of the Scattered Disk. Of course, particles scattered by Neptune also tend to spend a large fraction of their time near resonances due to the resonance sticking phenomenon (e.g., Duncan \& Levison 1997; Malyshkin \& Tremaine 1999). Therefore, the discrimination between scattered and resonantly trapped particles must be done carefully. Toward this end, we examine the orbital histories of all surviving particles in the shaded zone in Figure 4 that have $e>0.25$ and $a>50 \mathrm{AU}$. The usual definition of being "in" a $j+k: j$ mean-motion resonance is that the particle's resonance angle $\phi_{j k}$ (eq. [A1]) librates about some fixed value with some modest amplitude $\left|\Delta \phi_{j k}\right|$ that is usually $\lesssim 90^{\circ}$, while the resonance angle for a scattered body that is temporarily "stuck" in a resonance will have a $\phi_{j k}$ that circulates over $\pm 180^{\circ}$. However, we find that $\left|\Delta \phi_{j k}\right|$ is not the best discriminant for identifying trapped and scattered particles, because a small but significant fraction of particles do get trapped at a resonance with a $\phi_{j k}$ that is either circulating or else librating with a very large amplitude. For some trapped particles, this distinction is unclear due to this simulation's infrequent time sampling, which occurs every $\Delta t=10^{8} \mathrm{yr}$.

Rather, a more reliable discriminant between trapped and scattered particles is based on Brouwer's integral $B$ (eq. [A6]). This integral is conserved by resonantly trapped particles but is not conserved by scattered particles that are temporarily exhibiting the resonance sticking phenomenon. Of the 134 par-

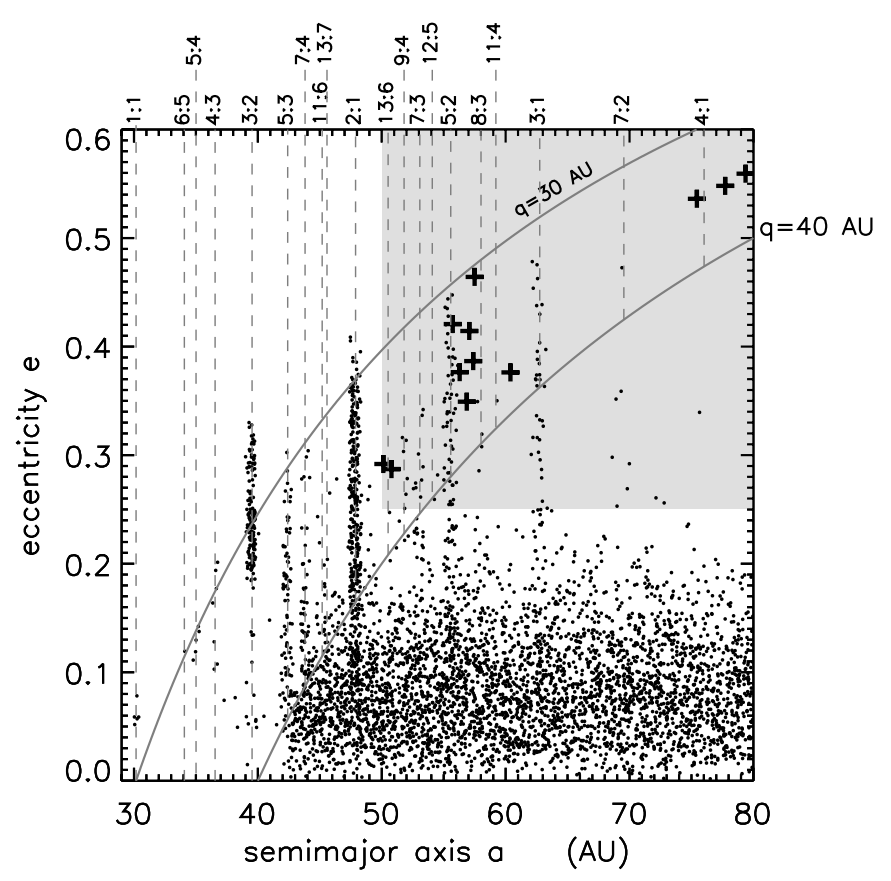

Fig. 4. -Eccentricity $e$ vs. semimajor axis $a$ at time $t=4.5 \times 10^{9} \mathrm{yr}$ for the model population with initial $e \sim 0.1$ shown in Fig. 3. The vertical dashed lines indicate the mean-motion resonances occupied by trapped particles with perihelia $q<40 \mathrm{AU}$ or particles inhabiting resonances in the gray region with libration amplitudes $\left|\phi_{j k}\right| \leq 90^{\circ}$. Crosses indicate scattered particles.

ticles that inhabit the gray zone in Figure 4, only 12, or about $10 \%$ of these particles, are truly scattered particles whose orbits $(a, e)$ evolve stochastically about the $30 \mathrm{AU} \lesssim q \lesssim 40$ AU zone; these scattered particles are indicated by the crosses in Figure 4. The remaining particles are resonantly trapped particles, most ${ }^{3}$ of which preserved their $B$-integral to within $\sim 3 \%$.

The orbits of all particles having perihelia $q<40$ AU have also been inspected, and those resonances inhabited by trapped particles having libration amplitudes $\left|\phi_{j k}\right| \leq 90^{\circ}$ are indicated by the vertical dashed lines in the figure. We find that particles get trapped at a number of exotic resonances, such as 11:6, $13: 7,13: 6,9: 4,12: 5,8: 3$, and $11: 4$.

\section{KUIPER BELT INCLINATIONS}

Inspection of the inclinations shown in Figure 3 suggests that the smooth migration model does not produce sufficient numbers of bodies in high-inclination orbits. This has been recognized in previous studies (Malhotra 1995; Gomes 1997) but has not been quantified. However, one should not directly compare the simulation's $i$-values to the observed KBO inclinations, since the latter are heavily biased by telescopic selection effects. Note that most telescopic surveys of the Kuiper Belt observe near the ecliptic, which favors the discovery of lower $i$ KBOs that spend a larger fraction of their time at lower latitudes (Jewitt \& Luu 1995). To mitigate this selection effect, one should instead consider the ecliptic inclination distribution, which is the inclination distribution of objects having latitudes $\beta$ very near the ecliptic (e.g., Brown 2001). The ecliptic inclination distribution

\footnotetext{
${ }^{3}$ However, the $e$ - and $i$-values of some resonantly trapped particles will still oscillate with constant $a$ in a manner that preserves their Jacobi integral; this evolution usually occurs after migration has ceased, and these particular motions do not preserve $B$.
} 


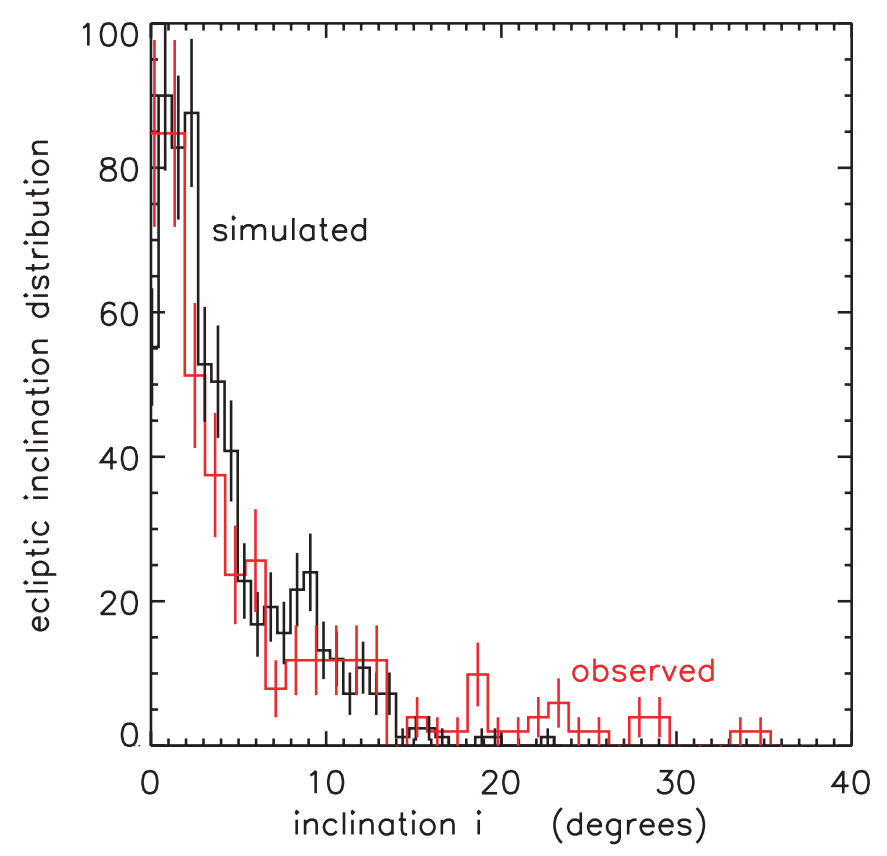

FIG. 5.-Ecliptic inclination distributions for the simulated and observed bodies in Fig. 3 that have perihelia $q<42 \mathrm{AU}$ and latitudes $|\beta| \leq 1^{\circ}$. Error bars give the Poisson counting uncertainty, and the vertical scale is arbitrary.

for multiopposition $\mathrm{KBOs}$ having perihelia $q \leq 42 \mathrm{AU}$ and latitudes $|\beta| \leq 1^{\circ}$ is shown in Figure 5 (red curve), as well as the simulated ecliptic inclination distribution for particles from Figure 3 that are selected similarly (black curve).

The agreement seen in Figure 5 among bodies having inclinations of $0^{\circ} \lesssim i \lesssim 15^{\circ}$ shows that the smooth migration model can readily recover the Kuiper Belt's lower inclination members. Of course, this agreement is partly due to the particles' initial inclinations being distributed around $\langle i\rangle \simeq 3^{\circ}$. But Figure 5 also shows this model to be quite deficient in producing sufficient numbers of the high- $i$ bodies having $i \gtrsim 15^{\circ}$. Similar results are also obtained among bodies orbiting at Neptune's 3:2 resonance. This is a serious discrepancy, since Brown (2001) has shown that there are two inclination populations in the Kuiper Belt: a minor population of low $i$ having characteristic inclinations of $i \sim 3^{\circ}$, and a high- $i$ population having $i \sim 15^{\circ}$, containing about three-quarters of all KBOs. Note that these high- $i$ bodies are very underrepresented in Figure 5 due to telescopic selection effects.

Of course, Neptune-scattered particles routinely achieve high inclinations of $i \gtrsim 15^{\circ}$; for instance, many of the high- $i$ particles seen in Figure 2 were scattered by Neptune. Could the Scattered Disk be a source of the high- $i$ KBOs that are found elsewhere in the Belt? Recent $N$-body simulations by Gomes (2003) show that a small fraction of these Neptune-scattered particles can evolve from very eccentric, Neptune-crossing orbits into less eccentric orbits in the Main Belt. In Gomes's simulations, this occurs principally at secular and mean-motion resonances that drive large oscillations in a scattered particle's eccentricity. When a scattered particle visits a resonance, it can have its $e$ temporarily lowered and its $q$ raised. If this occurs during the planet-migration epoch, this process becomes irreversible and can strand scattered particles in the Main Belt with high inclinations. Such bodies are identified by Gomes as "evaders," since they are Neptune-crossing bodies that ultimately manage to evade Neptune when deposited in the Main Belt. Note, however, that the efficiency of this process is quite low, affecting only $\epsilon \sim 0.002$ of all scattered particles in the simulation, which is evolved over the age of the solar system by Gomes (2003). However, all the high-i particles seen in our simulation (Fig. 3) achieved their inclinations while temporarily or permanently trapped in Neptune's advancing resonances. There were no Neptune-scattered evaders having $i>10^{\circ}$ that survived in our simulations.

Despite the evader mechanism's inefficiency, a model can still be constructed that yields a KBO inclination distribution that is quite similar to the observed one. For instance, this can be achieved by making the number density of small bodies initially orbiting interior to $\sim 27 \mathrm{AU}$ about 60 times higher than the density of bodies initially orbiting beyond 27 AU. As Gomes (2003) shows, Neptune's migration through this densely populated inner disk creates the Kuiper Belt's high- $i$ evaders, while the sparse outer disk provides the Belt's low- $i$ component. Although this scheme yields an $i$-distribution that does indeed agree with the observations, that success is achieved via a special configuration of the initial particle disk.

However, Levison \& Morbidelli (2003) avoid this problem of special initial conditions by assuming that the initial planetesimal disk simply ended at $\sim 35 \mathrm{AU}$. This is the "push-out" model, which argues that most of the Kuiper Belt is a consequence of Neptune's advancing 2:1 resonance, which can drag bodies outward to litter the Main Belt with low- $i$ KBOs. The Belt's high- $i$ component is then presumed to be due to the evader mechanism. While the push-out model remains quite intriguing, it would be interesting to see this scenario subjected to greater scrutiny to see whether it can indeed reproduce the Kuiper Belt's curious mix of high- and low-inclination KBOs in a self-consistent manner.

\section{A KUIPER BELT CENSUS: COMPARISON WITH OBSERVATIONS}

Figure 6 plots the relative abundance, over time, of the simulated Belt's various dynamical classes among particles having perihelia $q \leq 45 \mathrm{AU}$. These curves are normalized such that the final abundance of the Main Belt, where 40.1 AU $\leq$ $a \leq 47.2 \mathrm{AU}$, is unity. Note that this model predicts a $2: 1$ resonance that is 1.4 times more abundant than the Main Belt and 2.5 times more abundant than the $3: 2$ resonance, while the observations (Fig. 3) show a 2:1 resonance that is only sparsely populated. Of course, when comparing the simulated population to the observed population, one must first deal with the observational selection effect that strongly favors the discovery of larger and/or nearer KBOs. However, it is shown below that the effects of this bias can be accounted for by using a Monte Carlo method that assigns random sizes to the simulated population. This then allows one to make a fair comparison of the relative abundances of the simulated and observed populations.

Begin by letting $N(R)$ be the number of bodies in the simulated population having radii exceeding a radius $R$. Also, let $\xi$ be a random number that is uniformly distributed between 0 and 1 and interpret this number as the probability of selecting a body with a radius that is smaller than $R$. This is also equal to the probability of not selecting a body of radius $>R$, so $\xi=1-$ $N(R) / N_{\text {tot }}$, where $N_{\text {tot }}$ is the total number of bodies in this population. Since most small-body populations have a cumulative size distribution $N(R)$ that varies as a power law, adopt

$$
N(R)=N_{\text {tot }}\left(\frac{R}{R_{\min }}\right)^{-Q},
$$




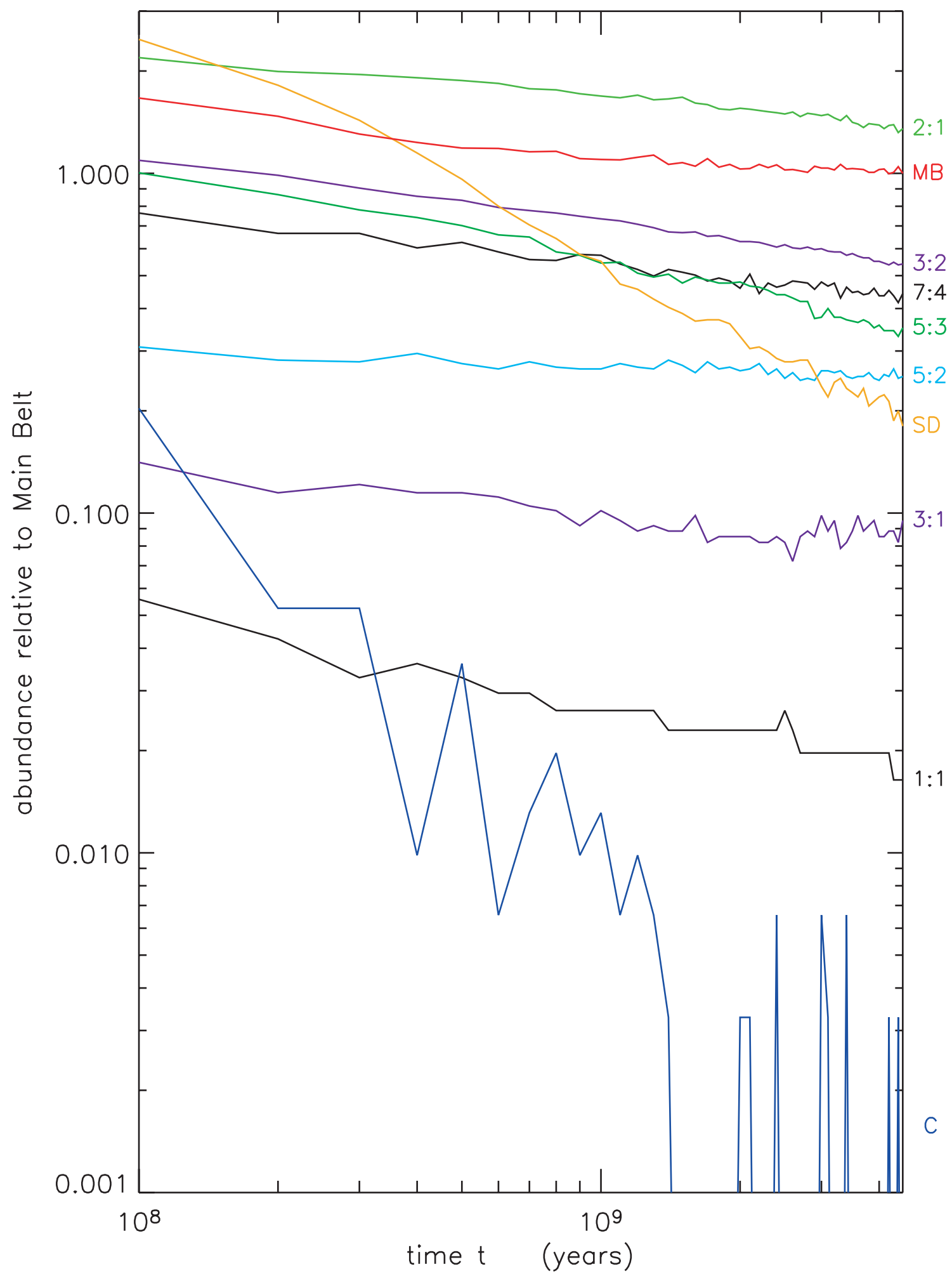

FIG. 6.-Abundances of the Belt's various dynamical populations plotted over time $t$, with all populations normalized to the Main Belt (line MB) population at time $t=4.5 \times 10^{9} \mathrm{yr}$ and counting only those particles with perihelia $q \leq 45 \mathrm{AU}$. The resonant populations are counts of all particles with semimajor axes within $0.6 \mathrm{AU}$ of exact resonance, while the Main Belt curve gives the number of particles with semimajor axes $40.1 \mathrm{AU} \leq a \leq 47.2 \mathrm{AU}$, excepting bodies in or near the 5:3 and 7:4 resonances. However, the Scattered Disk (line $S D$ ) curve shows the number of particles with $a>49$ AU and perihelia $q<40$ AU that are not members of any of the indicated resonances (e.g., the 5:2 and 3:1), while Centaurs (line $C$ ) are particles with $a$ less than Neptune's semimajor axis. 
where $R_{\min }$ is the radius of the smallest member of the swarm. Then $R / R_{\min }=(1-\xi)^{-1 / Q}$, but $1-\xi$ can be replaced with $\xi$, since these random numbers have the same distribution, so

$$
R(\xi)=\frac{R_{\min }}{\xi^{1 / Q}}
$$

Equation (5) is then used to generate random sizes for the simulated population of Figure 3 , which has apparent $R$-band magnitudes of

$$
m_{R}=m_{\odot}-2.5 \log \left[p\left(\frac{R}{r_{1}}\right)^{2}\left(\frac{r}{r_{1}}\right)^{-2}\left(\frac{r-r_{1}}{r_{1}}\right)^{-2}\right],
$$

where $r$ is the particle's heliocentric distance, $r_{1}=1 \mathrm{AU}$, $m_{\odot}=-27.29$ is the Sun's apparent $R$ magnitude, $R_{\min }=20 \mathrm{~km}$ is adopted here, and the observation is presumed to occur at solar opposition. All our calculations also adopt the usual albedo of $p=0.04$, so that our findings can be readily compared to past results obtained by others. However, if an alternate albedo $p$ is desired, simply revise all $\mathrm{KBO}$ radii reported here by a factor of $(p / 0.04)^{-1 / 2}$ and all masses by a factor of $(p / 0.04)^{-3 / 2}$. Finally, note that a power-law size distribution results in a cumulative luminosity function that varies as $\Sigma(m) \propto 10^{\alpha m}$, where $\Sigma(m)$ is the sky-plane number density of KBOs brighter than apparent magnitude $m$, and the logarithmic slope is $\alpha=$ Q/5 (Irwin et al. 1995).

Hubble Space Telescope (HST) observations reveal that the bright end of the Kuiper Belt's luminosity function has a steep logarithmic slope of $\alpha=0.88 \pm 0.1$ for bodies having magnitudes $m_{R} \ll 24$, while the faint end $\left(m_{R} \gg 24\right)$ of the luminosity function has a shallow logarithmic slope of $\alpha=0.32 \pm 0.15$ (Bernstein et al. 2004); the steeper slope of the bright end of the luminosity function was also confirmed recently by Elliot et al. (2005). This luminosity function can be interpreted as evidence that the KBO size distribution is actually two power laws that break even at a magnitude $m_{\mathrm{br}} \simeq 24$, which corresponds to a body of radius $R_{\mathrm{br}} \simeq 65 \mathrm{~km}$ orbiting at a characteristic distance of $r \sim 40 \mathrm{AU}$, assuming it has an albedo of $p=0.04$. However, our application concentrates only on those KBOs that have known orbits, and $99 \%$ of those bodies have magnitudes $m_{R}<$ $m_{\text {br }}$. Consequently, this study is sensitive only to the larger end of the KBO size spectrum, and such bodies are characterized here via a single power-law size distribution having $\alpha=0.88$ and $Q=5 \alpha=4.4$.

Although $\sim 5000$ simulated particles in Figure 3 manage to survive over the age of the solar system, the Monte Carlo model assigns far too few of them with sizes that would be detected by any telescopic survey of the Kuiper Belt. To boost the statistics of the detectable portion of the simulated population, each survivor is replicated $10^{4}$ times, such that each particle's orbital elements $a, e, i, \Omega$, and $\omega$ are preserved, while its mean anomaly $M$ is randomly distributed over $0 \leq M<2 \pi$. It should be noted that this step effectively assumes that the particles' longitudes are uniformly distributed over $2 \pi$, which is not quite correct since Neptune's resonant perturbations tend to arrange the particles' longitudes in a nonuniform manner (Malhotra 1996; Chiang \& Jordan 2002). Nonetheless, this is not a major concern since the observed KBOs were discovered along lines of sight that are distributed roughly uniformly in ecliptic longitude, which effectively washes out Neptune's azimuthal arrangement of the Belt; see Appendix $\mathrm{C}$ for a more detailed examination and justification of this assumption. Finally, this Monte Carlo model is then tested by verifying that the randomly generated population does indeed exhibit the expected luminosity function, which varies as $\Sigma(m) \propto 10^{Q m / 5}$.

Further comparison of the Monte Carlo model of the Kuiper Belt to any observations must be done carefully. Note that the brighter KBOs tend to be discovered in shallow, wide-angle surveys that observe a large area $\Delta \Omega$ on the sky, while the fainter KBOs tend to be discovered in deeper surveys that observe smaller areas $\Delta \Omega$. Consequently, the observed abundances of the various KBO subclasses (e.g., the Main Belt and the Scattered Disk) are proportional to all of these surveys' total area $\Omega\left(m_{R}\right)$, which itself is some function of the limiting magnitude $m_{R}$. However, Appendix B shows that this dependence on $\Omega\left(m_{R}\right)$ can be factored out by constructing ratios of the Belt's various subclasses. Appendix B also shows that the ratio of the observed abundance of any two dynamical classes of KBOs is approximately equal to the ratio of the intrinsic abundances of the much larger unseen populations. ${ }^{4}$ Thus, by plotting ratios of the simulated populations to the observed KBO populations, we can compare the model to the observations in a manner that is insensitive to survey details such as individual sky coverage $\Delta \Omega$.

Figure 7 shows the apparent abundances of the $2: 1$ and the $3: 2$ populations relative to the Main Belt (MB) as a function of their $R$-band magnitudes $m_{R}$. In the top panel, the light gray curve is the simulated ratio, which predicts an apparent $2: 1$ abundance of about $80 \%$ of the Main Belt, while the dark gray curve is the observed ratio. Taking the ratio of these two curves reveals that their discrepancy at magnitudes $m_{R}>21$ (which refers to about $90 \%$ of the observed subpopulations) is a factor of $f_{2: 1} \simeq 20$; the observed 2:1 resonance is markedly underabundant relative to the observed Main Belt population. There are $N_{\mathrm{MB}}(R>50 \mathrm{~km})=$ $1.0 \times 10^{5}$ Monte Carlo particles in the simulated Main Belt having radii $R>50 \mathrm{~km}$, and $N_{2: 1}(R>50 \mathrm{~km})=8.2 \times 10^{4}$ particles in the $2: 1$ resonance. If we let $r_{2: 1 / \mathrm{MB}}$ represent the inferred ratio of 2:1 to Main Belt objects, then $r_{2: 1 / \mathrm{MB}} \simeq N_{2: 1} / f_{2: 1} N_{\mathrm{MB}} \simeq 0.041$, which is comparable (albeit lower by a factor of $\sim 2$ ) to the ratio that Trujillo et al. (2001) infer from telescopic surveys of the Kuiper Belt.

The observed 3:2/MB ratio plotted in Figure 7 (bottom) also shows that this resonant population is underabundant relative to model predictions by a factor of $\sim 6$ among bright objects with $21 \lesssim m_{R} \lesssim 23$, and by a factor of $\sim 60$ at fainter magnitudes. Close inspection of the observations suggests that there is indeed a deficiency of fainter KBOs in the 3:2 resonance and that this curve is not due to some overabundance of Main Belt KBOs having magnitudes of $m_{R} \gtrsim 23$.

It should be noted that the results given in Figure 7 are not particularly sensitive to the detailed location of the Main Belt's outer edge. For instance, if we assume the Belt's primordial edge was instead at $a=45$ AU (e.g., Trujillo \& Brown 2001; see also $\S 7)$, this reduces both the $2: 1$ and Main Belt populations by about $40 \%$, while leaving the $3: 2$ population unchanged. Consequently, the $2: 1 / \mathrm{MB}$ ratios of Figure 7 are largely unchanged for both the simulated and observed populations, while the $3: 2 / \mathrm{MB}$ ratios increase by a factor of $(1-0.4)^{-1} \simeq 1.7$. However, the discrepancy between the simulated and observed populations is still the same factors of $\sim 6-60$.

\footnotetext{
${ }^{4}$ Of course, this method of analyzing the Belt's relative abundances will tell us little about those KBO populations that are either too rare, too dim, or otherwise too difficult to recover in telescopic surveys. Nonetheless, we still can use our method to place upper limits on the abundances of any hypothetical $\mathrm{KBO}$ populations that are unseen using the method described in $\S 7$.
} 

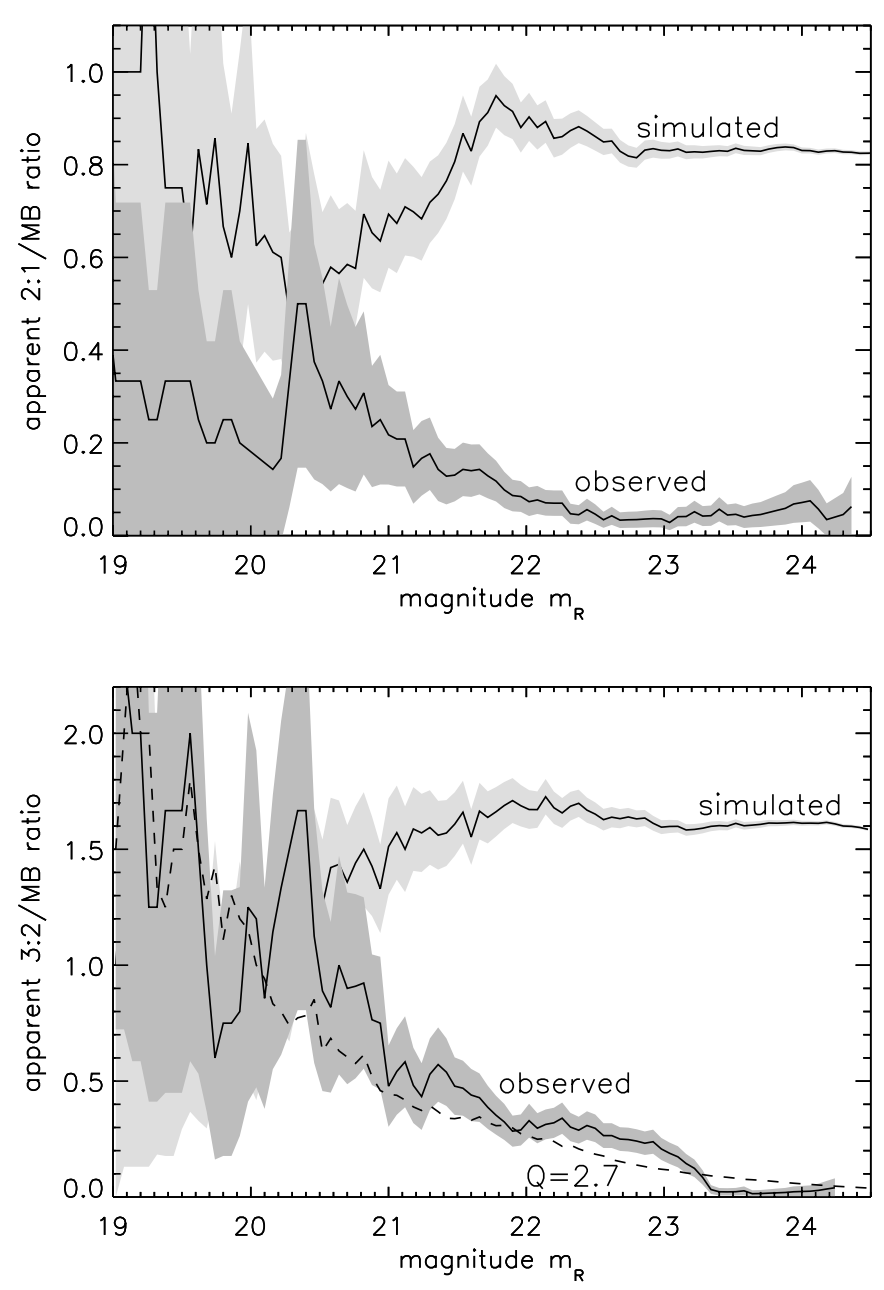

FIG. 7.- Number of KBOs that have been observed (for two or more oppositions) orbiting in or near the 2:1 resonance with apparent magnitudes $m_{R} \pm \Delta m$ relative to the observed Main Belt population at $40.1 \mathrm{AU} \leq a \leq 47.2 \mathrm{AU}$ (top, dark-gray curve), and the observed 3:2/MB ratio (bottom, dark-gray curve). Likewise, the light-gray curves show the 2:1/MB and 3:2/MB ratios for the simulation of Fig. 3 , in which these bodies' sizes and magnitudes are assigned using the Monte Carlo method of $\S 6$ assuming $Q=4.4$ and $R_{\min }=20 \mathrm{~km}$. All these curves are smoothed over a magnitude window with a half-width $\Delta m=0.5$, and the vertical half-widths of the gray zones are $1 \sigma$ assuming Poisson counting uncertainties. The observed curves end at $m_{R} \simeq 24.5$, which is the magnitude of the faintest multiopposition KBO. The dashed curve shows the simulated $3: 2 / \mathrm{MB}$ ratio when the $3: 2$ population has a shallow size distribution with $Q=2.7$ and $R_{\min }=4.3 \mathrm{~km}$, while the Main Belt bodies have $Q=4.4$ and $R_{\min }=20 \mathrm{~km}$.

Although there are several possible interpretations of the discrepancies seen in Figure 7, the most plausible explanation is that other unmodeled processes are responsible for (1) reducing the trapping efficiencies of the 2:1 and 3:2 resonances by factors of $\sim 6-60$ or (2) causing trapped particles to diffuse out of the resonances and into nearby regions of phase space that are quite unstable (see Fig. 1 of Duncan et al. 1995), resulting in their ejection from the Kuiper Belt. Such unmodeled processes include the collisions and gravitational scatterings that occurred with ever greater vigor during earlier times when the Belt was more crowded. The scattering of these planetesimals by Neptune was of course responsible for driving that planet's migration, so the occasional scattering of a large and/or close planetesimal will cause that planet's orbit and hence its resonances to shudder some. Likewise, scattering events among the KBOs themselves would also cause their semimajor axes to diffuse some, as would collisions. This means that scatterings and collisions will have driven a random walk in the resonant particles' semimajor axes, as well as a random walk in the locations of the resonances themselves. It is possible then that these unmodeled effects can drive particles out of resonances and reduce the resonant population by the large factors indicated in Figure 7, a scenario that is also explored in simulations by Zhou et al. (2002).

The magnitude dependence of the observed $3: 2 / \mathrm{MB}$ ratio shown in Figure 7 is also quite curious. The fact that the observed ratio varies with apparent magnitude $m_{R}$, while the simulated ratio remains constant at magnitudes fainter than $m_{R}=$ 21 , suggests that the $N(R) \propto R^{-Q}$ power law that was universally applied throughout the entire Belt is overly simplistic. One way for the model to achieve better agreement with the observations is to assume that larger, brighter bodies are more abundant in the $3: 2$ resonance and that smaller, fainter bodies are less abundant there than they are in the Main Belt, which requires a shallower size distribution. The dashed curve in Figure 7 illustrates this possibility, which shows the simulated $3: 2 / \mathrm{MB}$ ratio assuming that the $3: 2$ bodies have a shallow $Q=2.7$ size distribution with $R_{\min }=4.3 \mathrm{~km}$ (note that reducing $R_{\min }$ has the effect of reducing the total number of visible objects), while the Main Belt bodies have the usual distribution with $Q=4.4$ and $R_{\min }=20 \mathrm{~km}$. The shallow size distribution that is inferred here for the $3: 2$ population is also consistent with the logarithmic slope of $\alpha \simeq 0.56$ that Elliot et al. (2005) recently reported for the luminosity function of their "resonant" population, which is dominated by $3: 2 \mathrm{KBOs}$; the size distribution inferred from that work is $Q=5 \alpha=2.8$. KBO sizes can also vary with inclination (Levison \& Stern 2001, but see also footnote 1 of Gomes 2003). In particular, Bernstein et al. (2004) report that the bright end of the luminosity function for highinclination $\left(i>5^{\circ}\right) \mathrm{KBOs}$ has a logarithmic slope of $\alpha=0.66$ and a size distribution $Q=5 \alpha=3.3$, which is much shallower than the low- $i$ KBOs with $\alpha=1.36$ and a $Q=5 \alpha=6.8$.

In our Monte Carlo model there are only $N_{3: 2}(R>50 \mathrm{~km})=$ 2100 bodies larger than $R=50 \mathrm{~km}$, so their numerical abundance relative to the Main Belt is $r_{3: 2 / \mathrm{MB}}=N_{3: 2} / N_{\mathrm{MB}}=0.021$, which again is comparable (but again lower by a factor of $\sim 2$ ) to the ratio reported in Trujillo et al. (2001). The largest Monte Carlo body at the $3: 2$ resonance has a radius $R \simeq 1000 \mathrm{~km}$, which is comparable to the size of the largest multiopposition KBO there ${ }^{5}$ with $R \simeq 1100 \mathrm{~km}$, assuming $p=0.04$.

This range of power-law indices that is inferred for the Kuiper Belt, $2.7 \lesssim Q \lesssim 4.4$, is comparable to the values of $Q$ that are observed at various sites throughout the asteroid belt. NearEarth objects have a fairly shallow size distribution with $Q=$ 1.95 (Stuart \& Binzel 2004), while the asteroid families exhibit steeper size distributions. For instance, Figure 1 of Tanga et al. (1999) shows size distributions for several prominent asteroid families having values of $2 \lesssim Q \lesssim 6$. Note also that nonfamily asteroids have $Q \simeq 3.0$ (Ivezić et al. 2001), which is slightly steeper than the canonical $Q=2.5$ value that results from a collisional cascade (Dohnanyi 1969). Since the various asteroid subclasses exhibit such a wide variation in their size distributions over a relatively narrow range of semimajor axes of $\Delta a \sim$ $4 \mathrm{AU}$, perhaps it should be of no surprise that the spatially much wider Kuiper Belt might also exhibit some variety in $Q$.

\section{THE OUTER EDGE OF THE SOLAR SYSTEM}

Inspection of Figure 3 shows a prominent absence of observed KBOs having modest eccentricities of $e \sim 0.1$ near and

\footnotetext{
${ }^{5}$ Excepting Pluto, of course, which has an anomalously high albedo of $p \sim 0.5$.
} 


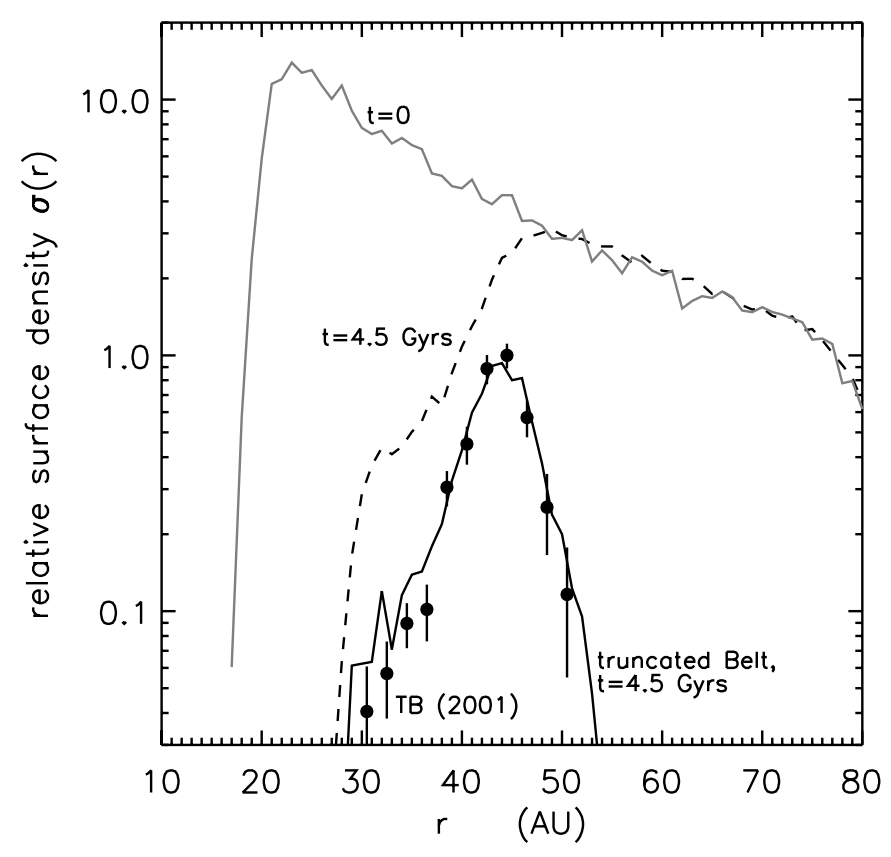

FIG. 8.-KBO surface number density $\sigma(r)$ (circles), normalized to peak at unity and inferred from the $\mathrm{KBO}$ radial distribution function $f(r) \propto r \sigma(r)$ reported by Trujillo \& Brown (2001). The $t=0$ and 4.5 Gyr curves show the simulated Belt's initial and final surface densities. The "truncated Belt" curve shows the simulation's final surface density assuming that the Belt is truncated at $a=45 \mathrm{AU}$ and that the $3: 2$ population also does not contribute to $\sigma$. Note that the surface density of the inner half of this Belt increases as a very steep function of distance, $\sigma(r) \propto r^{9.5}$ for $r \lesssim 45 \mathrm{AU}$.

beyond Neptune's 2:1 resonance. The prevailing interpretation of this observed feature is that there is a boundary near 45 AU $\lesssim$ $a \lesssim 50$ AU that marks the outer edge of the solar system's primordial Kuiper Belt (Allen et al. 2001; Trujillo \& Brown 2001). The circles in Figure 8 show the Belt's surface density $\sigma(r)$ inferred from these observations, which peaks at $r \simeq 45 \mathrm{AU}$. We have nonetheless allowed our simulated Kuiper Belt to extend out to $a=80 \mathrm{AU}$ in order to use the dearth of observed distant KBOs to place quantitative upper limits on the abundance of hypothetical KBOs that might live beyond $50 \mathrm{AU}$.

The $N$-body/Monte Carlo model of $\S 6$ can be used to predict how many KBOs should have been observed in the $a>50 \mathrm{AU}$ zone (which we identify here as the Outer Belt [OB]) assuming (1) that the primordial Kuiper Belt extends smoothly out to $a=$ 80 AU and (2) that all KBOs everywhere have the same size distribution with the usual parameters $Q=4.4$ and $R_{\min }=20 \mathrm{~km}$. This simulation's ratio of Outer Belt to Main Belt objects, $r_{\mathrm{OB} / \mathrm{MB}}\left(m_{R}\right)$, is plotted versus magnitude $m_{R}$ in Figure 9. This is the ratio of $N_{\mathrm{OB}}^{\mathrm{sim}}\left(m_{R}\right)$, the number of bodies in the simulated Outer Belt (whose members have semimajor axes 50 AU $<a<$ $80 \mathrm{AU}$ and eccentricities $e<0.2)$, to $N_{\mathrm{MB}}^{\mathrm{sim}}\left(m_{R}\right)$, the number of bodies in the simulated Main Belt (where 40.1 AU $<a<$ 47.2 AU) in the magnitude interval $m_{R} \pm \Delta m$, where $\Delta m=$ 0.5 . According to the figure, the expected $\mathrm{OB} / \mathrm{MB}$ ratio is $r_{\mathrm{OB} / \mathrm{MB}}=0.4$. At present there are $N_{\mathrm{MB}}^{\mathrm{obs}}=264 \mathrm{KBOs}$ in the Main Belt that have been observed for two or more oppositions, and the dimmest member of this group of KBOs has an apparent magnitude $m_{R}^{*}=24.5$. The $N$-body/Monte Carlo model thus predicts that there should also be $N_{\mathrm{OB}}^{\mathrm{obs}}=r_{\mathrm{OB} / \mathrm{MB}} N_{\mathrm{MB}}^{\mathrm{obs}} \simeq 100$ objects brighter than $m_{R}^{*}$ orbiting in the Outer Belt beyond $a=50 \mathrm{AU}$. This prediction is in marked contrast with the observations, which show that there are no known multiopposition objects orbiting in the Outer Belt with magnitudes brighter than $m_{R}^{*}$, i.e., $N_{\mathrm{OB}}^{\mathrm{obs}}<1$.

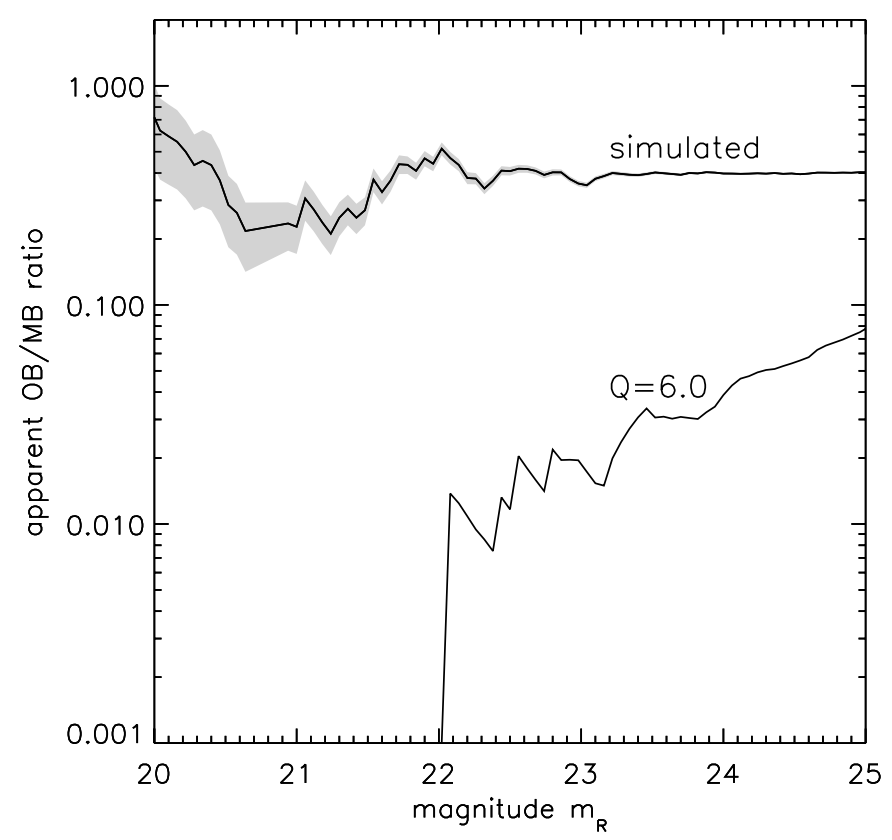

FIG. 9.-Expected ratio of bodies in the Outer Belt $(a>50 \mathrm{AU})$ relative to the Main Belt (40.1 AU $<a<47.2 \mathrm{AU}$ ) as a function of magnitude $m_{R}$ assuming the size distribution has $Q=4.4$ and $R_{\min }=20 \mathrm{~km}$ ( gray curve). The black curve shows the $\mathrm{OB} / \mathrm{MB}$ ratio assuming the Outer Belt instead has a steeper $Q=6.0$ size distribution, while the Main Belt has $Q=4.4$, with $R_{\min }=20 \mathrm{~km}$ for both populations.

This prediction that the Outer Belt would have an observed abundance that is $40 \%$ of the Main Belt differs considerably from that of Gladman et al. (1998), who estimated that the observed Outer Belt population should only be $\sim 6 \%$ of the total observed population. However, this much lower estimate was obtained by assuming that the current Belt's surface number density resembles its primordial $\sigma(r)$, which likely varied as $r^{-2}$ or so. This very common assumption causes the inner part of the Belt to be more concentrated than its outer part. However, a real Kuiper Belt would have been dynamically eroded over the eons by the giant planets' gravitational perturbations. This dynamical erosion is illustrated in Figure 8, which shows the simulated Belt's primordial surface density ( gray curve) and its final surface density (dashed curve); similar erosion is also seen in the longterm integrations of Duncan et al. (1995). Figure 8 shows that $\sigma(r)$ for an eroded Belt is a sharply increasing function of $r$ for $r \lesssim 50 \mathrm{AU}$, which implies that the inner observable portion of the Belt is very underdense relative to the more distant $r \sim 50 \mathrm{AU}$ zone. This dynamical erosion accounts for the discrepancies between our Outer Belt predictions and that by Gladman et al. (1998).

Recall that Figures 3 and 7 show that the 2:1 and 3:2 populations are very depleted relative to model predictions and that the zone beyond Neptune's 2:1 resonance is either empty or inhabited by bodies too small and faint to be seen. To account for these depletions, the solid curve in Figure 8 also shows a revised surface density curve obtained from the simulated Belt that is truncated at $a=45$ AU (about 3 AU inward of Neptune's 2:1 resonance), and with the negligible contribution from the $3: 2$ population also being ignored. This results in a curve that agrees quite well with the Belt's observed surface density variations. Despite this good agreement in the radial distributions of the simulated and observed Kuiper Belts, Figure 3 shows that this apparent edge at $a=45 \mathrm{AU}$ is still rather fuzzy, since there are four multiopposition KBOs of low eccentricity $(e<0.05)$ orbiting in the Main Belt at 45 AU $<a<48$ AU. Close inspection of Figure 3 shows that a hard edge at $a=45$ AU also could not account for 
the KBOs having $e \sim 0.1$ in the 45 AU $<a<48$ AU zone, unless the advancing 2:1 resonance also dragged some bodies out of the $a<45$ AU zone and deposited them here, reminiscent of the scenario suggested by Levison \& Morbidelli (2003).

The remainder of this section places upper limits on the size and abundance of any unseen KBOs that might lurk beyond $a=50$ AU. Of course, there are multiple interpretations of the dearth of observed multiopposition bodies orbiting beyond $a=$ 50 with modest eccentricities of $e \sim 0.1$, i.e., that $N_{\mathrm{OB}}^{\mathrm{obs}}<1$. One interpretation of this upper limit is that assumption 1 is incorrect - that the primordial Kuiper Belt's density did not extend smoothly beyond Neptune's 2:1 resonance but instead was reduced by a factor $f$ (relative to the smooth model's density) in the $a>50 \mathrm{AU}$ zone. In this case, the $\mathrm{OB} / \mathrm{MB}$ ratio becomes

$$
r_{\mathrm{OB} / \mathrm{MB}}=0.4 / f=N_{\mathrm{OB}}^{\mathrm{obs}} / N_{\mathrm{MB}}^{\mathrm{obs}}<1 / N_{\mathrm{MB}}^{\mathrm{obs}}
$$

which implies that the primordial density of the Outer Belt was smaller than the Main Belt by a factor $f \gtrsim 100$.

Alternatively, assumption 2 could be incorrect, namely, it could be that the KBO size distribution is not uniform everywhere. For instance, the absence of any multiopposition bodies in the Outer Belt having magnitudes brighter than $m_{R}^{*}=24.5$ could simply mean that bodies beyond $r \simeq 50 \mathrm{AU}$ are dimmer than $m_{R}^{*}$ and thus have radii smaller than $R \simeq 80(p / 0.04)^{-1 / 2} \mathrm{~km}$ (see eq. [6]). Note that Trujillo et al. (2001) obtained a similar limit but that they came to regard this scenario as unlikely.

It is also possible that the Outer Belt's size distribution is steeper, i.e., has a larger $Q$, than the Main Belt's size distribution. An increase in $Q$ decreases the abundance of bright bodies, as is illustrated by the curve in Figure 9, which gives the $r_{\mathrm{OB} / \mathrm{MB}}$ ratio for an Outer Belt having a $Q=6.0$ size distribution, while bodies in the Main Belt have the usual $Q=4.4$ distribution. This particular $Q$ is also the minimum value consistent with the observed upper limit of $r_{\mathrm{OB} / \mathrm{MB}}<1 / 264$; Outer Belts with a smaller $Q$ would contain at least $1 \mathrm{KBO}$ brighter than $m_{R}^{*}$ in the $a>50 \mathrm{AU}$ zone for every $264 \mathrm{KBOs}$ detected in the Main Belt, while an Outer Belt having a larger $Q$ would be undetected. This particular model is near the threshold of detection, and its largest member has a radius of $R=250 \mathrm{~km}$. Trujillo et al. (2001) also considered this scenario, but they concluded that the absence of distant KBOs requires a steeper $Q>9$ size distribution. The origin of this discrepancy is unclear.

It is also interesting to note that the low-inclination KBOs have a logarithmic slope of $\alpha=1.36$ along the bright end of their luminosity function (Bernstein et al. 2004), which implies a steep size distribution of $Q=5 \alpha=6.8$. Such bodies, if they inhabit the Outer Belt beyond $a=50$ AU with the same abundances as adopted by our model, could conceivably have avoided detection to date due to their steep size distribution. In other words, a Main Belt whose low- $i$ population extends beyond $a=$ 50 AU while its high- $i$ population terminates at $a=50$ AU could be quite consistent with their nondetection.

From these considerations it can be concluded that the observed absence of multiopposition KBOs in the $a>50$ AU zone having modest eccentricities $e \sim 0.1$ implies (1) this part of the primordial Kuiper Belt was underdense by a factor $f \gtrsim 100$ relative to the $a<50$ AU zone, (2) these distant KBOs have radii $R \lesssim 80 \mathrm{~km}$, (3) their size distribution has a power-law index $Q>6.0$, or perhaps (4) some combination of the above effects.

\section{THE ORIGIN OF CENTAURS}

It is generally accepted that Centaurs are those bodies that have diffused inward from the Kuiper Belt into orbits that cross

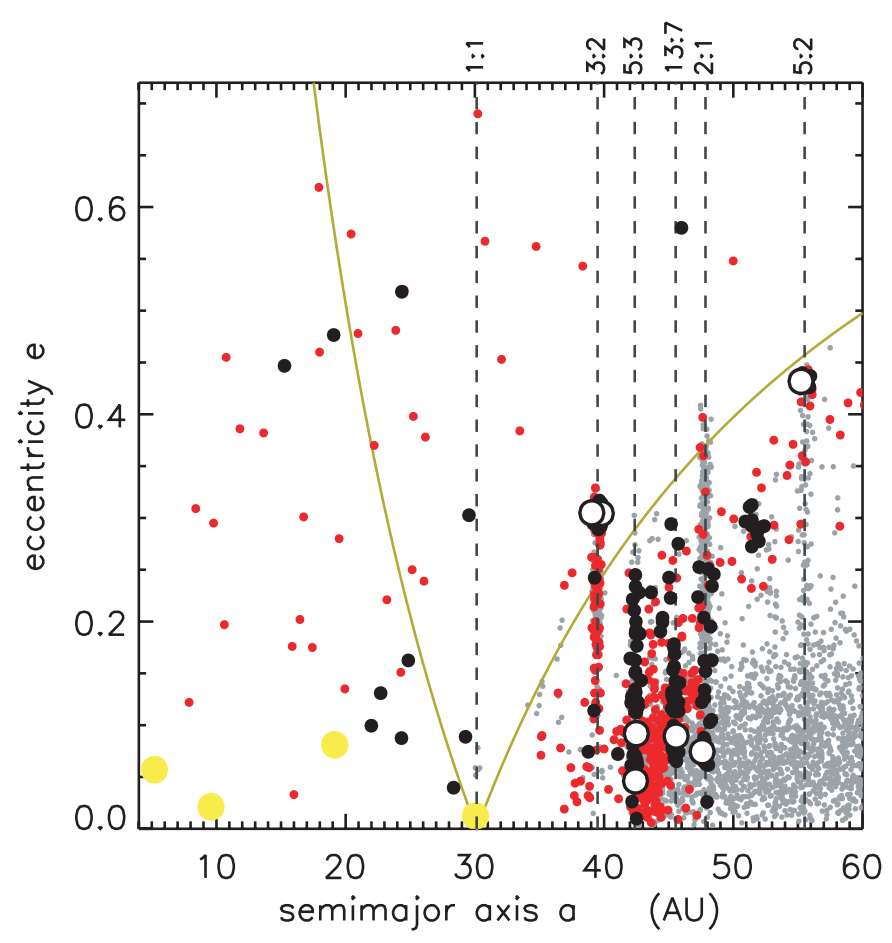

FIG. 10.-Orbits of the simulation's seven Centaurs at time $t=10^{8} \mathrm{yr}$ (open circles) and their subsequent motions (black circles). Gray circles show the simulation's end state at time $t=4.5 \times 10^{9} \mathrm{yr}$, red circles indicate multiopposition KBOs and Centaurs, and yellow circles show the giant planets' final orbits. Neptune's mean-motion resonances are indicated, and the curve is the threshold for Neptune-crossing orbits.

the giant planets (e.g., Duncan et al. 1988). Currently there are 27 known Centaurs observed for more than one opposition; these are the red circles in Figure 10 having $a<30$ AU. Since planet crossers are quickly ejected or accreted, Centaurs have short dynamical lifetimes of only $\sim 10^{7} \mathrm{yr}$ (Levison \& Duncan 1997; Tiscareno \& Malhotra 2003). Consequently, the density of these "escapees from the Kuiper Belt" (e.g., Stern \& Campins 1996 ) is very tenuous inside $a=30$ AU (see Fig. 8). Indeed, only $N_{\mathrm{C}}^{*}=7$ Centaurs are detected during the final $\Delta T=$ 2 billion yr of our simulation, which was only sparsely time sampled once every $\Delta t=10^{8} \mathrm{yr}$, so the instantaneous number of Centaurs is $N_{\mathrm{C}}=N_{\mathrm{C}}^{*}(\Delta t / \Delta T)=0.35$ at the end of the simulation. There are also $N_{\mathrm{MB}}=565$ bodies in the Main Belt, so the Centaur/Main Belt ratio is provisionally estimated at $r_{\mathrm{C} / \mathrm{MB}}=6.2 \times 10^{-4}$.

The open circles in Figure 10 show the orbital elements of these seven Centaurs at time $t=10^{8} \mathrm{yr}$, which is at a time when planet migration has only recently ceased. Thus, the open circles indicate the locations where Neptune has parked these proto-Centaurs in the Kuiper Belt. Note that all seven Centaurs originate from sites in or near Neptune's mean-motion resonances, namely, the $3: 2,5: 3,13: 7,2: 1$, and $5: 2$. Their subsequent motions at times $t>10^{8} \mathrm{yr}$ are shown as black circles (again, poorly time sampled), which show that the eccentricities of nearly all proto-Centaurs initially wander up and down with constant $a$ until they have a close encounter with Neptune, scatter off that planet, make a brief appearance in the $a<$ 30 AU Centaur zone, and then are quickly removed from the system.

These seven bodies have initial semimajor axes of $28 \mathrm{AU} \leq$ $a \leq 48 \mathrm{AU}$ at time $t=0$, so Centaurs can also be regarded as samples that have been drawn from a wide swath of the outer 
solar nebula. Figure 10 also shows that the simulated Centaurs are concentrated just inside Neptune's orbit; their mean heliocentric distance is $r=26 \pm 3 \mathrm{AU}$, and their mean inclination is $i=16^{\circ} \pm 10^{\circ}$. Note also that three of the seven Centaurs emerged from the 3:2 and 2:1 resonances, which $\S 6$ showed to be heavily depleted relative to the model's predictions. Consequently, the Centaur/Main Belt ratio reported above should instead be interpreted as an upper limit, e.g., $r_{\mathrm{C} / \mathrm{MB}}<6.2 \times 10^{-4}$. It is shown in $\S 11$ that our model predicts that there are $N_{\mathrm{MB}} \simeq$ $1.3 \times 10^{5}$ Main Belt KBOs having radii $R>50 \mathrm{~km}$, so this model also predicts that there are $N_{\mathrm{C}}=r_{\mathrm{C} / \mathrm{MB}} N_{\mathrm{MB}}<80$ similarly sized Centaurs.

Although the Centaur upper limit reported here is comparable to the population that Sheppard et al. (2000) infer from the Centaur luminosity function, there is still a prominent disconnect in the heliocentric distances of the simulated and observed populations; our simulated Centaurs all reside at $r>$ 22 AU, while the three Centaurs that Sheppard et al. (2000) used to construct the Centaur luminosity function were detected at heliocentric distances of $r<19$ AU. One possible interpretation of this excess of Centaurs at $r \lesssim 20$ AU is that Centaurs may be breaking up and spawning new Centaurs (e.g., Pittich \& Rickman 1994) as they wander among the giant planets. Finally, we note that deep, wide-angle surveys of the Kuiper Belt, such as the Legacy Survey that is currently being implemented at the CanadaFrance-Hawaii Telescope, may soon reveal the existence of the Centaurs anticipated by this model to reside at greater distances of 23 AU $\lesssim r \lesssim 29$ AU.

\section{NEPTUNE'S TROJANS}

Figure 3 also shows that $N_{\mathrm{T}}=5$ particles managed to survive the length of the simulation at Neptune's 1:1 resonance. These simulated particles are, of course, Neptune's Trojans, of which two are currently known: $2001 \mathrm{QR}_{322}$ (Chiang et al. 2003) and $2004 \mathrm{UP}_{10}$ (Sheppard et al. 2005). For this simulation the Trojan/ Main Belt ratio is $r_{\mathrm{T} / \mathrm{MB}}=N_{\mathrm{T}} / N_{\mathrm{MB}}=8.8 \times 10^{-3}$, where $N_{\mathrm{MB}}=$ 565 is the number of survivors that persist in the Main Belt. The spatial coordinates of the two observed and five simulated Trojans are shown in Figure 11, which indicates that these particles can roam about with longitudes $\pm 30^{\circ}$ from Neptune's triangular Lagrange points and semimajor axes $\pm 0.32 \mathrm{AU}$ from Neptune's. The extent of these Trojan sites is similar to that seen in integrations by Holman \& Wisdom (1993) and Nesvorný $\&$ Dones (2002). Note that no special effort was made to start any of the simulated particles at Neptune's Lagrange points. Rather, all particles were distributed randomly about a disk according to a smooth surface density law, with the inner edge of the disk being well inside Neptune's initial tadpole region. In our simulation the five survivors had initial semimajor axes approximately \pm 0.28 AU from Neptune's initial $a$, and there were a total of 68 particles initially in Neptune's Trojan source region (i.e., $|\Delta a| \leq 0.28 \mathrm{AU}$ and $|\Delta \phi| \leq 30^{\circ}$ ), so the surviving Trojan fraction is about $7 \%$. This survival fraction is comparable to that obtained by Kortenkamp et al. (2004) in a similar simulation. That work also showed that as planets migrate, several secondary resonances sweep across the 1:1 resonance, which results in a heavy loss of Neptune's Trojans during the migration epoch.

Neptune's Trojans are of interest since they might place constraints on some models of the early evolution of the outer solar system. For example, Thommes et al. (1999, 2002) postulate that Neptune originally formed in the vicinity of Jupiter and Saturn and was tossed outward after scattering off the larger planets. But the existence of $2001 \mathrm{QR}_{322}$ and $2004 \mathrm{UP}_{10}$ might cast doubt on this scenario, since Trojans seem unlikely to per-

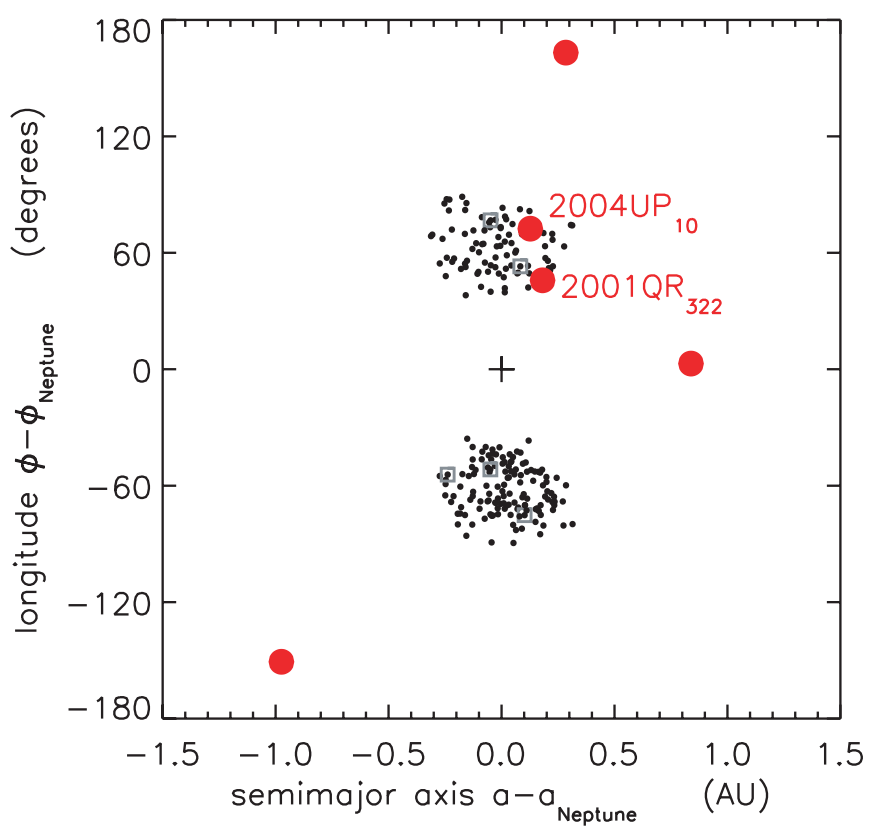

FIG. 11.-Longitudes offive simulated Trojans vs. their semimajor axes (black circles), all relative to Neptune's and sampled every $10^{8} \mathrm{yr}$ during the entire simulation. Neptune lies at the plus sign. Small squares show the final positions of these Trojans, indicating that two Trojans lie at the leading Lagrange point, with three trailing. Red circles indicate three nearby "field" KBOs, as well as Neptune's two known Trojans, $2001 \mathrm{QR}_{322}$ and $2004 \mathrm{UP}_{10}$.

severe at Neptune's Lagrange points during such a scattering event. However, it has since been shown that a recently scattered Neptune can still acquire its Trojans later as its orbit is circularized by a dense Kuiper Belt (H. F. Levison 2005, private communication). It is also conceivable that Neptune may have captured its Trojan from a heliocentric orbit after Neptune's orbit had settled down. Although Kortenkamp et al. (2004) show that the direct capture of Trojans from heliocentric space is rare and results in only transient Trojans, Chiang \& Lithwick (2005) show that mutual collisions can insert small bodies into stable orbits at Neptune's Lagrange points.

\section{THE EXTENDED SCATTERED DISK}

Figure 12 shows the orbits of those scattered particles that have been tossed into very wide orbits about the Sun. Most of the simulated scattered particles have perihelia between 30 and $40 \mathrm{AU}$, as do most of the observed scattered KBOs. However, there are two exceptions to this rule, namely, $2000 \mathrm{CR}_{105}$ and $2003 \mathrm{VB}_{12}$ (also known as Sedna), which have respective perihelia of $q=$ $44.14 \pm 0.02$ (Gladman et al. 2002) and $q=76 \pm 4$ AU (Brown et al. 2004). Gladman et al. (2002) classify those Scattered Disk KBOs having perihelia higher than $q \simeq 40$ AU as members of the so-called Extended Scattered Disk. Sedna's large radius of $R \sim 1000 \mathrm{~km}$ makes this object a particular curiosity, since its discovery circumstances suggest that there may be a few hundred other unseen Sedna-sized objects (Brown et al. 2004). Since Sedna has a mass of $\sim 10^{-3} M_{\oplus}$, the implied mass that might be hidden in the Extended Scattered Disk is a few tenths of an Earth mass. Thus, Sedna by itself may represent an enormous reservoir of unseen mass that is comparable to the "conventional" Kuiper Belt (see $\S 11)$.

The Extended Scattered Disk is also of dynamical interest since, as Gladman et al. (2002) note, many dynamical models of the Kuiper Belt (including this one) generally produce Scattered 


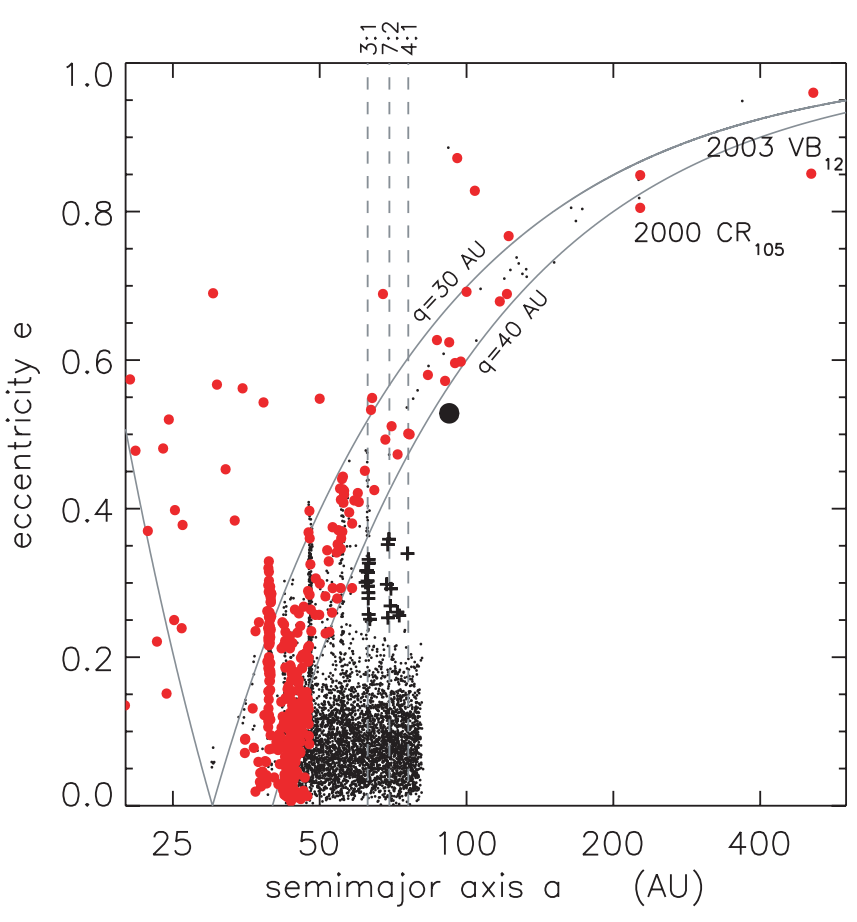

FIG. 12.-Eccentricities $e$ vs. $a$ on a logarithmic axis for the particles of Fig. 3. Red circles show the KBOs observed over multiple oppositions, and the curves indicate the $q=40 \mathrm{AU}$ threshold, as well as Neptune-crossing orbits. Two members of the so-called Extended Scattered Disk, $2000 \mathrm{CR}_{105}$ and 2003 $\mathrm{VB}_{12}$, are also indicated. Crosses indicate particles resonantly trapped at the $3: 1$, $7: 2$, and $4: 1$ resonances. The large black circle indicates the only simulated particle that was scattered into a high-perihelion orbit $(q=43.5 \mathrm{AU})$, resembling that of $2000 \mathrm{CR}_{105}$.

Disk objects in lower perihelia orbits having $q \lesssim 40$ AU. Gladman et al. (2002) review a number of scenarios that might explain how a $\mathrm{KBO}$ might get promoted from a nearly circular orbit into a wide, eccentric orbit having $q \gtrsim 40 \mathrm{AU}$; these include (1) chaotic diffusion of scattered bodies, (2) gravitational scattering by long-gone massive protoplanets, (3) scattering by an undiscovered distant planet, and (4) scattering by a single star that passes within $\sim 100 \mathrm{AU}$ of the Sun. However, all these scenarios are problematic. For instance, billion-year integrations of $\sim 10^{4}$ particles in chaotic Neptune-scattered orbits fail to diffuse into orbits having perihelia as high as that of $2000 \mathrm{CR}_{105}$ (Gladman et al. 2002). Morbidelli et al. (2002) also cast doubt on scenarios 2 and 3 by showing that $\sim 20 \%$ of any distant population of protoplanets would have persisted over the age of the solar system, and that some fraction of these large bodies should already have been discovered by one of the various wide-angle Kuiper Belt surveys. Scenario 4 is also in doubt, since simulations of a close encounter with a single star generally produce disturbances in the outer Kuiper Belt that are quite unlike that seen in the observed Belt (e.g., Ida et al. 2000). However, Fernández \& Brunini (2000) have shown that repeated encounters with more distant stars can produce Sedna-like orbits. This may have occurred early, while the Sun was still a member of the open cluster from which it presumably formed. In this scenario, the giant planets scatter small bodies into wide orbits of $a \sim 100-1000 \mathrm{AU}$, which the nearby cluster stars then perturb into Sedna-like orbits having higher perihelia. Scattering by a passing star was recently reexamined by Morbidelli \& Levison (2004), and their simulations also support this scenario.

Although our simulations did not produce any Sedna-like objects in orbits that are well decoupled from the giant planets, we did find a single scattered object in a $2000 \mathrm{CR}_{105}$-like orbit in the Extended Scattered Disk with a semimajor axis of $a=92 \mathrm{AU}$ and a perihelion of $q=43.5 \mathrm{AU}$ (Fig. 12, large black circle). The orbital history of this scattered particle is shown in Figure 13, which shows that as the particle inhabited Neptune's 16:3 resonance during $2 \mathrm{Gyr} \lesssim t \lesssim 3 \mathrm{Gyr}$, some process raised this Scattered Disk particle's perihelion up and into the Extended Scattered Disk on a billion-year timescale. This kind of behavior was first reported in Levison \& Duncan (1997) and Duncan \& Levison (1997), whose simulations also show that some scattered particles can achieve high perihelia orbits while in or near mean-motion resonances.

However, we have not identified any particular resonance as being responsible for raising the perihelia of our one $\mathrm{CR}_{105}$ candidate shown in Figure 13. For instance, a Kozai resonance is not implicated since the argument of perihelion $\omega$ does not librate. The possibility of other Pluto-like "super-resonances" (e.g., Malhotra \& Williams 1997) was also examined; this is the libration of a resonance angle of the form $\phi_{j k}=j\left(\omega-\omega_{\mathrm{N}}\right)-k(\Omega-$ $\Omega_{\mathrm{N}}$ ), where $\Omega$ is the particle's longitude of the ascending node and the " $N$ " subscript refers to Neptune's orbital elements. Angles having $0 \leq|j|,|k| \leq 10$ were examined, and although the angle $\phi_{j k}=5\left(\Omega-\Omega_{\mathrm{N}}\right)$ did in fact librate for about $1 \mathrm{Gyr}$, that occurred well after the time when the particle's $q$ was raised. A resonance involving interactions with multiple planets is also unlikely, since the particle's Tisserand parameter $T$ (which is simply its Jacobi integral sans the interaction energy due to Neptune) was well preserved during these times. Although the particular mechanism that drove this particle into the Extended Scattered Disk is not understood, this particle does demonstrate that it is indeed possible for Scattered Disk particles to diffuse into the Extended Scattered Disk via planetary perturbations alone, with other external agents (such as stellar encounters) being absent. This transport from the Scattered Disk to the Extended Scattered Disk via mean-motion resonances is also evident in the simulations of Levison \& Duncan (1997) and Duncan \& Levison (1997). However, this transport has an extremely low flux, since only one of the $\sim 5 \times 10^{3}$ particles initially in the Scattered Disk did manage to enter the Extended Scattered Disk and persist over the age of the solar system. External perturbations from passing stars (Fernández \& Brunini 2000; Morbidelli \& Levison 2004) may indeed be more effective at producing members of the Extended Scattered Disk.

Note also the simulated particles represented by crosses in Figure 12. Even though their perihelia of $42 \mathrm{AU}<q<54 \mathrm{AU}$ might suggest that they also inhabit the domain of the Extended Scattered Disk, they are in fact resonant particles that were trapped at the $3: 1,7: 2$, and $4: 1$ resonances during the migration epoch. Most of these particles have libration amplitudes less than $\left|\Delta \phi_{j k}\right| \lesssim 90^{\circ}$. These particles also had initial semimajor axes of $a>47 \mathrm{AU}$, which is noteworthy since, if any resonant KBOs are ever discovered in these orbits, they could be interpreted as evidence that the outer edge of the solar system lies beyond $a>47 \mathrm{AU}$. However, that interpretation would still be ambiguous, since Neptune-scattered evaders, which originated from smaller semimajor axes, can also settle into these same resonances (see Fig. 18 of Gomes [2003] for an example).

\subsection{The Scattered Disk}

Figure 14 shows the apparent abundance of so-called Scattered Disk (SD) objects relative to the Main Belt, as predicted by the $N$-body/Monte Carlo model. There are $N_{\mathrm{SD}}=1.9 \times 10^{4}$ Monte Carlo bodies in orbits having $50 \mathrm{AU}<a<150 \mathrm{AU}$ and perihelia $28 \mathrm{AU}<q<40 \mathrm{AU}$, while $N_{\mathrm{MB}}=1.0 \times 10^{5}$ Monte Carlo particles survive in the Main Belt, so the model predicts an 


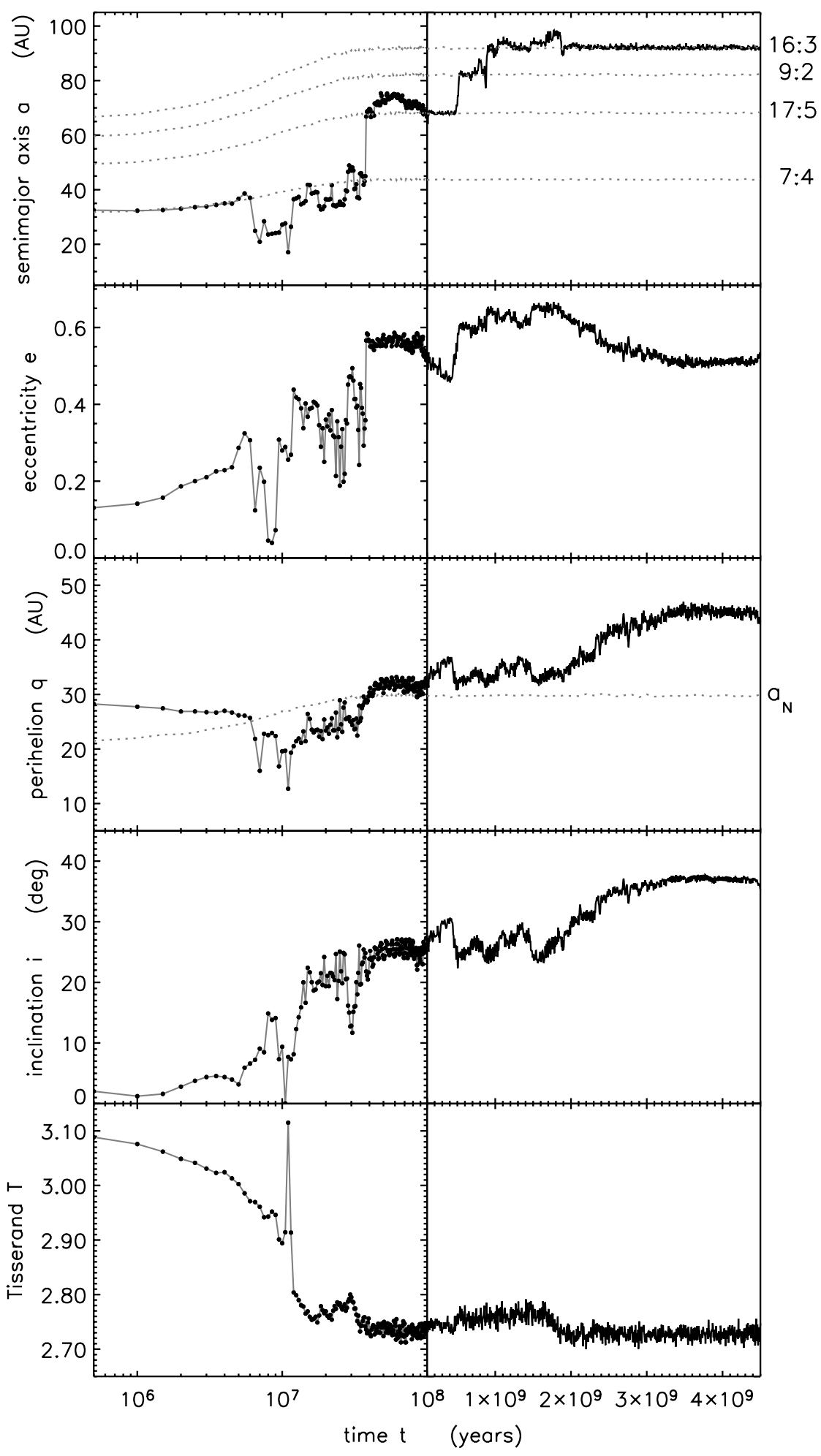

FIg. 13.-Orbital history of the only simulated scattered object that inhabits the Extended Scattered Disk (e.g., the large black circle in Fig. 12). The particle's $a, e, q$, $i$, and Tisserand parameter $T=a_{\mathrm{N}} / a+2 \sqrt{\left(a / a_{\mathrm{N}}\right)\left(1-e^{2}\right)} \cos i$ are first plotted vs. logarithmic time (left panels) and then linearly (right panels). The dotted curves show Neptune's semimajor axis $a_{\mathrm{N}}$ and a few of its mean-motion resonances. 


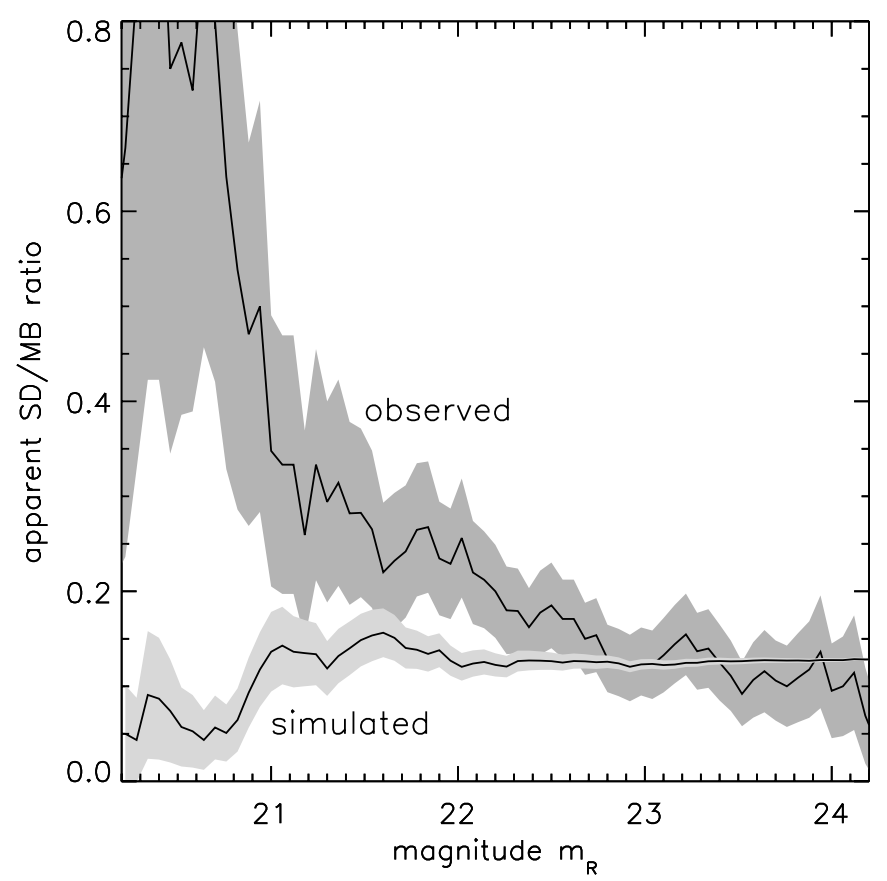

FIg. 14.-Observed ratio of Scattered Disk objects, in orbits with $50 \mathrm{AU}<$ $a<150 \mathrm{AU}$ and perihelia $28 \mathrm{AU}<q<40 \mathrm{AU}$, relative to Main Belt objects vs. apparent magnitude $m_{R}$ (dark-gray curve). The light-gray curve is the simulated ratio.

intrinsic $\mathrm{SD} / \mathrm{MB}$ ratio of $r_{\mathrm{SD} / \mathrm{MB}}=N_{\mathrm{SD}} / N_{\mathrm{MB}}=0.19$. The apparent ratio of these two populations is $\sim 0.1$, also shown in Figure 14. Note that the intrinsic SD/MB ratio inferred here is about one-fourth that reported in Trujillo et al. (2001).

\section{CALIBRATION}

The ecliptic luminosity function of Bernstein et al. (2004) is shown in Figure 15, and its bright end varies as $\Sigma\left(m_{R}\right)=$ $10^{\alpha\left(m-m_{0}\right)} \mathrm{deg}^{-2}$, where $\alpha=0.88$ and $m_{0}=23.1$ for magnitudes $m_{R}<m_{\mathrm{br}}=24$. This luminosity function gives the number density of KBOs near the ecliptic that are brighter than magnitude $m_{R}$. Since this curve scales with the total number of KBOs, it can be used to calibrate the simulation to determine the total number of objects in the Kuiper Belt.

Sections 6 and 7 show that the observed 3:2 and 2:1 populations are severely depleted relative to model predictions and that bodies in the Outer Belt beyond the 2:1 resonance are either absent or too faint to be seen. To account for these depletions, a truncated Kuiper Belt similar to that of $\S 7$ is adopted; this Belt is formed by discarding any bodies orbiting beyond the 2:1 resonance, as well as all bodies orbiting within $\Delta a=0.6 \mathrm{AU}$ of Neptune's 3:2 and 2:1 resonances. There are $N_{N \text {-body }}=587$ $\mathrm{N}$-body particles in this truncated Kuiper Belt, and they are replicated $N_{\text {reps }}=10^{4}$ times with sizes and magnitudes assigned to them according to the Monte Carlo method of $\S 6$, with $Q=4.4$ and $R_{\min }=20 \mathrm{~km}$.

The simulation's median inclination is low (e.g., $\S 5$ ), only $\bar{i}_{\text {sim }}=2.7$, which is much lower than the median inclination $\bar{i}_{\text {obs }}=15^{\circ} .6$ that is inferred from the debiased KBO inclination distribution reported by Brown (2001). Due to these low inclinations, the simulation's ecliptic luminosity function would thus be artificially overdense by a factor $f_{i}=\bar{i}_{\mathrm{obs}} / \bar{i}_{\mathrm{sim}} \simeq 5.8$, so it is revised downward by this factor to compensate. The simulation's $\Sigma\left(m_{R}\right)$ is then multiplied by a factor $f_{\Sigma}=3.5$ to fit it to the bright end of the observed luminosity function; this ac-

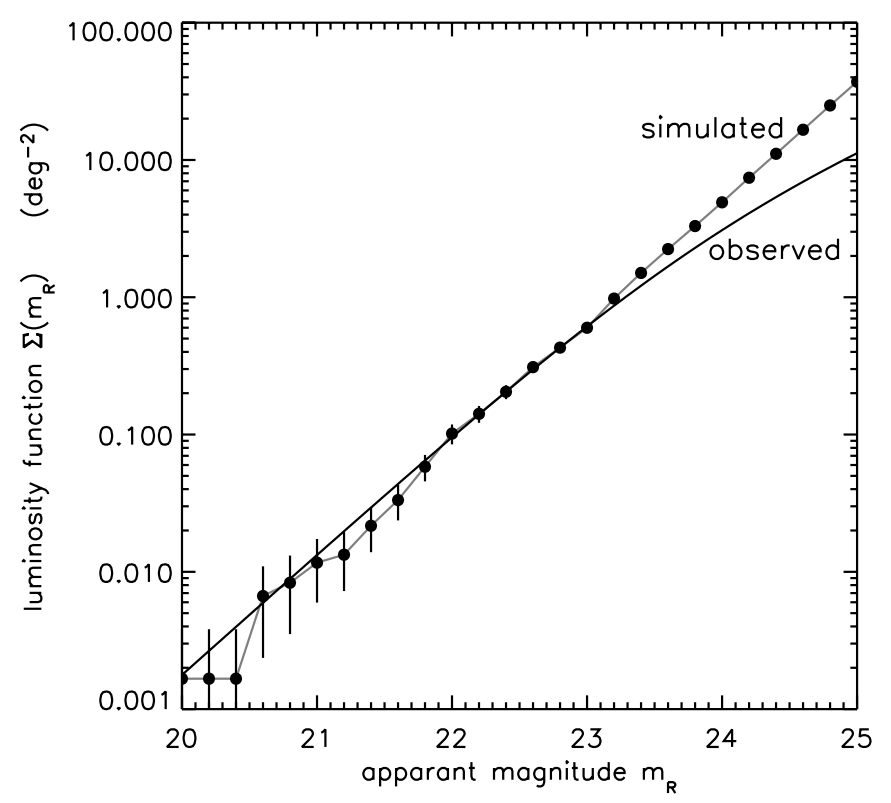

Fig. 15.-Kuiper Belt's observed ecliptic luminosity function $\Sigma\left(m_{R}\right)$ (smooth black curve), obtained by integrating the differential luminosity function $d \Sigma\left(m_{R}\right) /$ $d m_{R}$ reported in Bernstein et al. (2004). Note that it breaks to a shallower slope at magnitudes $m_{\mathrm{br}} \simeq 24$. The circles give the simulation's ecliptic luminosity function for particles in a truncated Kuiper Belt (see $\S 11$ ) with latitudes within 0.5 of the ecliptic. Error bars are for Poisson counting statistics.

counts for the different populations in the simulated and observed Kuiper Belts and results in the curve shown in Figure 15. The size distribution adopted here is valid down to a radius of about $R_{\mathrm{br}}=65 \mathrm{~km}$ (see $\S 6$ ), so the inferred number of KBOs larger than $R_{\mathrm{br}}$ is

$$
N_{\mathrm{br}}=N_{N \text {-body }} N_{\text {reps }} f_{\Sigma}\left(R_{\mathrm{br}} / R_{\text {min }}\right)^{-Q} \simeq 1.1 \times 10^{5} .
$$

To estimate the total number of KBOs larger than the fiducial radius of $R_{50}=50 \mathrm{~km}$, note that the faint end of the observed luminosity function has a logarithmic slope of $\alpha_{\text {faint }}=0.32$ (Bernstein et al. 2004), which implies a power-law index of $Q_{\text {faint }}=5 \alpha_{\text {faint }}=1.6$ for bodies having radii $R<R_{\mathrm{br}}$. The total number of bodies larger than $R_{50}$ is thus

$$
N_{50}=N_{\mathrm{br}}\left(R_{50} / R_{\mathrm{br}}\right)^{-Q_{\text {faint }}} \simeq 1.7 \times 10^{5} .
$$

The total mass of these bodies is obtained from their cumulative size distribution, which for the large bodies with $R>R_{\mathrm{br}}$ can be written $N(R)=N_{\mathrm{br}}\left(R / R_{\mathrm{br}}\right)^{-Q}$. The differential size distribution is then $d N(R)=|d N / d R| d R$, and if $M(R)$ is the mass of a body having a radius $R$, the total mass of bodies having radii in the interval $R_{\mathrm{br}}<R<R_{\max }$ is

$$
\begin{aligned}
M\left(R>R_{\mathrm{br}}\right) & =\int_{R_{\mathrm{br}}}^{R_{\max }} M\left(R^{\prime}\right) d N\left(R^{\prime}\right) \\
& =\frac{Q}{Q-3}\left[1-\left(\frac{R_{\mathrm{br}}}{R_{\max }}\right)^{Q-3}\right] N_{\mathrm{br}} M_{\mathrm{br}},
\end{aligned}
$$

where

$$
\begin{aligned}
M_{\mathrm{br}} & =M\left(R_{\mathrm{br}}\right) \\
& \simeq 1.2 \times 10^{21}\left(\frac{\rho}{1 \mathrm{~g} \mathrm{~cm}^{-3}}\right)\left(\frac{p}{0.04}\right)^{-3 / 2} \mathrm{~g},
\end{aligned}
$$


which is the mass of a body of radius $R_{\mathrm{br}}=65 \mathrm{~km}$ assuming it has a density $\rho$ and albedo $p$. The total mass of KBOs larger than $R_{\text {br }}$ with semimajor axes inside Neptune's 2:1 resonance is thus

$$
M\left(R>R_{\mathrm{br}}\right) \simeq 0.07\left(\frac{\rho}{1 \mathrm{~g} \mathrm{~cm}^{-3}}\right)\left(\frac{p}{0.04}\right)^{-3 / 2} M_{\oplus}
$$

for a $Q=4.4$ size distribution that extends to radii as large as $R_{\text {max }}=1000 \mathrm{~km}$. To get the total mass of bodies at the fiducial size $R=R_{50}$, add to the above the mass of bodies in the size interval $R_{50}<R<R_{\mathrm{br}}$, which is roughly $\Delta M \simeq\left(N_{50}-N_{\mathrm{br}}\right) M_{\mathrm{br}}$. The total mass of bodies larger than $R_{50}=50 \mathrm{~km}$ is then

$$
\begin{aligned}
M_{\mathrm{tot}} & =M\left(R>R_{\mathrm{br}}\right)+\Delta M \\
& \simeq 0.08\left(\frac{\rho}{1 \mathrm{~g} \mathrm{~cm}^{-3}}\right)\left(\frac{p}{0.04}\right)^{-3 / 2} M_{\oplus} .
\end{aligned}
$$

Note that the $0.08 M_{\oplus}$ prefactor is a consequence of adopting the oft-employed Halley albedo of $p=0.04$. However, recent observations indicate that KBOs have an average albedo of $p \simeq 0.1$ (Altenhoff et al. 2004; Grundy et al. 2005), which in turn lowers the Kuiper Belt mass to $M_{\text {tot }} \sim 0.02 M_{\oplus}$ assuming they have a unit density.

This population estimate is comparable to, but a bit higher than, previous estimates that rely on far simpler models of the Kuiper Belt. For instance, Trujillo et al. (2001) report a Main Belt population of $3.8 \times 10^{4}$ objects of mass $0.03 M_{\oplus}$ among bodies having radii $R>50 \mathrm{~km}$. They also estimate the Belt's total population to be 1.9 times the Main Belt population, so a total population of $N_{50} \sim 7.2 \times 10^{4}$ bodies larger than $R_{50}$ having mass $M_{\text {tot }} \simeq 0.06 M_{\oplus}$ is inferred. A similar estimate is also inferred from the HST survey by Bernstein et al. (2004); according to their Figure 8, the sky-plane number density of KBOs larger than $R_{50}$ is $\Sigma\left(R>R_{50}\right) \simeq 13 \mathrm{deg}^{-2}$. Since the Kuiper Belt subtends a total solid angle of $\Delta \Omega_{\text {tot }} \simeq 8100 \mathrm{deg}^{2}$ (Brown 2001), the total number of KBOs larger than $R_{50}$ is $N_{50}=\Sigma\left(R>R_{50}\right) \Delta \Omega_{\text {tot }} \simeq$ $1.1 \times 10^{5}$, with a total mass of $M_{\text {tot }} \simeq 0.05 M_{\oplus}$.

Sections 6-10 show that the simulated Belt's various dynamical classes have abundances of $r_{2: 1 / \mathrm{MB}}=0.041, r_{3: 2 / \mathrm{MB}}=$ $0.021, r_{\mathrm{C} / \mathrm{MB}}<6.2 \times 10^{-4}, r_{\mathrm{T} / \mathrm{MB}}<8.8 \times 10^{-3}$, and $r_{\mathrm{SD} / \mathrm{MB}}=$ 0.19 relative to the Main Belt, so the Main Belt fraction is $f_{\mathrm{MB}}=1-\sum_{i} r_{i / \mathrm{MB}} \simeq 0.74$; thus, there are $N_{\mathrm{MB}}(R>50 \mathrm{~km})=$ $f_{\mathrm{MB}} N_{50} \simeq 1.3 \times 10^{5}$ Main Belt KBOs having radii $R>50 \mathrm{~km}$. The numerical abundance of the $i$ th dynamical class is $N_{i}(R>$ $50 \mathrm{~km})=r_{i / \mathrm{MB}} f_{\mathrm{MB}} N_{50}$, and its mass is $M_{i}(R>50 \mathrm{~km})=$ $r_{i / \mathrm{MB}} f_{\mathrm{MB}} M_{\text {tot }}$, where $M_{\text {tot }}=0.08 M_{\oplus}$ assuming $\rho=1 \mathrm{~g} \mathrm{~cm}^{-3}$ and $p=0.04$; these abundances and masses are listed in Table 1. The exception is the $3: 2$ mass estimate, which adopts the $Q=$ 2.7 power-law size distribution described in $\S 6$; if this subgroup really does have such a flat size distribution, then equation (7) must be used to calculate its mass. ${ }^{6}$

Note also that the preceding ratios assume that the Main Belt terminates just inward of the $2: 1$ resonance at $a=47.2 \mathrm{AU}$. If, however, one wishes to adopt an outer edge at $a=45 \mathrm{AU}$, then $\S 6$ shows that this reduces the Main Belt population by $40 \%$, so that the ratios $r$ quoted above should then be raised by a factor of 1.7. The exception to this rule is the bodies at the 2:1 resonance; their abundance relative to the Main Belt is unchanged. However, the total number of KBOs reported here is

\footnotetext{
${ }^{6}$ With the quantities $R_{\mathrm{br}}, N_{\mathrm{br}}$, and $M_{\mathrm{br}}$ replaced by $R_{50}, r_{3: 2 / \mathrm{MB}} N_{\mathrm{MB}}$, and $M(R=50 \mathrm{~km})$.
}

\begin{tabular}{|c|c|c|c|}
\hline Dynamical Class & $\begin{array}{l}\text { Site } \\
(\mathrm{AU})\end{array}$ & Population & $\begin{array}{l}\text { Mass } \\
\left(M_{\oplus}\right)\end{array}$ \\
\hline Centaurs .................... & $a<a_{\mathrm{N}}$ & $<80$ & $<4 \times 10^{-5}$ \\
\hline 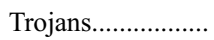 & $a=a_{\mathrm{N}}$ & $<1.1 \times 10^{3}$ & $<5 \times 10^{-4}$ \\
\hline $3: 2 \ldots \ldots \ldots \ldots \ldots \ldots \ldots$ & $a=39.5$ & $2.7 \times 10^{3}$ & $3.1 \times 10^{-3}$ \\
\hline Main Belt ...................... & $40.1 \leq a \leq 47.2$ & $1.3 \times 10^{5}$ & 0.059 \\
\hline 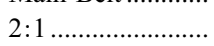 & $a=47.8$ & $5.3 \times 10^{3}$ & $2.4 \times 10^{-3}$ \\
\hline Scattered Disk..... & $50<a<150,28<q<40$ & $2.5 \times 10^{4}$ & 0.011 \\
\hline
\end{tabular}

TABLE 1

Abundance and Mass of KBOs with Radil from 50 to $1000 \mathrm{~km}$

Note.-Assuming density $\rho=1 \mathrm{~g} \mathrm{~cm}^{-3}$ and albedo $p=0.04$.

still insensitive to the detailed location of the Main Belt's outer edge, since that number is obtained by fitting the simulated KBOs' luminosity function to the observed $\Sigma\left(m_{R}\right)$, which is quite insensitive to the detailed location of the Main Belt's outer edge.

It should also be noted that this study employed an initial $\sigma(a) \propto a^{-2}$ disk surface density, but our findings are readily adapted for an alternate surface density law. For instance, if the canonical $\sigma(a) \propto a^{-1.5}$ law were instead desired, then this shallower power law would result in fewer objects trapped in the $3: 2$ resonance relative to the Main Belt population. Since the $3: 2$ objects are drawn from the $a_{3: 2} \sim 32$ AU part of the disk, while the Main Belt objects form at $a_{\mathrm{MB}} \sim 44 \mathrm{AU}$, this revised surface density law would reduce the $3: 2 / \mathrm{MB}$ ratio reported here by a factor $\left(a_{3: 2} / a_{\mathrm{MB}}\right)^{0.5} \sim 0.85$, which is a $15 \%$ change in relative abundance. Of course, the $2: 1 / \mathrm{MB}$ ratio would remain unchanged, since the source populations are the same.

Also, we conservatively interpret the abundance of Neptune's Trojans reported in Table 1 as an upper limit on their real abundance. It was argued in $\S 6$ that other unmodeled processes, possibly the scattering of planetesimals by Neptune or among themselves, would reduce the trapping efficiency of the $3: 2$ and 2:1 resonances by factors of $\sim 10$. Thus, it is possible that the same unmodeled phenomena might also have destabilized orbits at Neptune's 1:1 resonance, so the actual number of Trojan survivors may be smaller than that reported in $\S 11$.

Finally, upper limits on the abundance of KBOs inhabiting a hypothetical Outer Belt are reported for the $50 \mathrm{AU}<a<$ 80 AU zone assuming these bodies have the shallowest possible size distribution, namely, $Q=6.0$ down to $R_{\min }=20 \mathrm{~km}$ (see $\S 7$ ). In this case, there are at most $N_{\mathrm{OB}}=1.3 \times 10^{4}$ bodies in the Outer Belt having $50 \mathrm{~km}<R<250 \mathrm{~km}$ and a total mass of $M_{\mathrm{OB}} \simeq 0.008 M_{\oplus}$, assuming a density of $\rho=1 \mathrm{~g} \mathrm{~cm}^{-3}$ and an albedo $p=0.04$.

\section{EFFECTS NOT MODELED}

It should be noted that the model used here only accounts for the Belt's dynamical erosion that is a consequence of Neptune's gravitational perturbations; it does not account for the collisional erosion of the Kuiper Belt that is often invoked to account for the Belt's depleted appearance (e.g., Stern 1996; Kenyon \& Luu 1999). In particular, models of KBO accretion, as well as the self-consistent $N$-body simulations of Neptune's migration, all suggest that the Kuiper Belt's primordial mass was of order $\sim 30 M_{\oplus}$ (Stern 1996; Kenyon \& Luu 1999; Hahn \& Malhotra 1999; Gomes et al. 2004), which is at least 400 times more than the current mass. However, the model used here, which only accounts for the dynamical erosion, results in a depletion by a factor of about 3 in the $30 \mathrm{AU}<a<48$ AU zone of Figure 3 . This suggests that collisional erosion, which is not modeled here, may have been responsible for reducing the Belt's mass 
by an additional factor ${ }^{7}$ of $\sim 100$. Nonetheless, the abundance and mass estimates obtained here should still be reliable provided the Belt's collisional erosion was relatively uniform across the observable $35 \mathrm{AU} \lesssim a \lesssim 50$ AU zone. If, however, collisional erosion was more vigorous in some parts of the Belt, and less so in other parts, then the estimates obtained above are only accurate in an order-of-magnitude sense.

A comparable problem also occurs with the model's inclinations. Section 11 shows that the simulated Kuiper Belt is too thin by a factor $f_{i} \simeq 6$. This is compensated for by reducing the simulation's luminosity function $\Sigma\left(m_{R}\right)$ by the factor $f_{i}$, which is equivalent to increasing each particles' inclination by this factor. Again, this crude treatment should still yield a reliable estimate of the KBO population provided the factor $f_{i}$ is uniform everywhere and independent of semimajor axis $a$. If, however, $f_{i}$ were not independent of $a$, then this would result in errors in the relative abundances of the Belt's various subpopulations reported in Table 1.

We also note that the relative abundances of the Belt's various subpopulations are determined by a model that invokes a smooth outward migration by Neptune by $\Delta a_{\mathrm{N}} \simeq 9 \mathrm{AU}$, with the results reported in Figure 6 . That figure shows that the smooth migration scenario predicts a combined $3: 2+2: 1$ population that is comparable to the Main Belt population. This is because smooth migration is very efficient at trapping particles at Neptune's resonances, and this results in densely populated resonances. However, a detailed comparison of the model predictions to the observed abundances indicates that the resonant KBO population is really only about $5 \%$ of the Main Belt population (see $\S 6$, Table 1). The seemingly low abundance of resonant KBOs is likely due to unmodeled effects that may have occurred during the migration epoch, possibly due to the mutual scattering that might occur among bodies trapped at resonance, or perhaps due to the gravitational scattering of large planetesimals by Neptune (e.g., Zhou et al. 2002).

There is also evidence indicating that a wide swath of the early Kuiper Belt was stirred up prior to the onset of Neptune's migration. Recall that simulations of Neptune's outward migration into a dynamically cold Kuiper Belt are unable to account for the eccentricities of $e \sim 0.1$ observed among Main Belt KBOs $(\S 2)$. This suggests that the Belt was stirred up, either prior to or after the onset of Neptune's migration. However, $\S 3$ shows that this stirring event likely occurred prior to migration; migration into a stirred-up Kuiper Belt facilitates trapping at a multitude of weak, high-order mean-motion resonances, which, as Chiang et al. (2003) point out, is consistent with the detection of seven KBOs now known to librate at Neptune's 5:2 resonance.

It is then natural to ask what mechanism might be responsible for stirring up a broad swath of the Kuiper Belt, particularly since accretion models tell us that KBOs must have formed in a dynamically cold environment, i.e., the particles' initial $e$ - and $i$-values were $\lesssim 10^{-3}$ (Stern 1996; Kenyon \& Luu 1999). Note that this disturbance was probably not due to gravitational stirring by a number of long-gone protoplanets since, as Morbidelli et al. (2002) point out, a sizable fraction of such bodies would still persist in the Kuiper Belt and would likely have been discovered by now.

\footnotetext{
${ }^{7}$ Only a "conventional" Kuiper Belt model, such as the one explored here, need invoke additional erosion to reduce the Kuiper Belt mass by another factor of $\sim 100$. This is distinct from the push-out model, which need not rely on any collisional depletion of the Kuiper Belt (Levison \& Morbidelli 2003).
}

Note that this stirring mechanism must also have had a large reach, since it must have afflicted KBOs across the entire Main Belt, at least out to Neptune's 2:1 resonance. One mechanism that comes to mind is secular resonance sweeping, which is the only mechanism known to us that might stir eccentricities in the Belt up to $e \sim 0.1$ across its entire width (Nagasawa \& Ida 2000). Secular resonance sweeping is a consequence of the dispersal of the solar nebula gas; the removal of that gas alters the giant planets' precession rates, which in turn shifts the location of secular resonances (Ward 1981). The magnitude of the disturbance caused by a sweeping secular resonance depends on the timescale over which the nebula is depleted; longer depletion timescales $\tau_{\text {dep }}$ result in larger eccentricity pumping. The simulations of nebula dispersal by Nagasawa \& Ida (2000) show that a disturbance of $e \sim 0.1$ across much of the Kuiper Belt requires a nebula depletion timescale of $10^{6} \mathrm{yr} \lesssim \tau_{\text {dep }} \lesssim$ $10^{7}$ yr (but see also Hahn \& Ward 2002).

\section{DISCUSSION}

One of the goals of this study is to determine how the adoption of a particular Kuiper Belt model might affect an assessment of the Belt's total population and mass. Note that some models of the Belt assume that the KBOs are distributed according to a primordial surface density distribution that might vary with distance at $\sigma(r) \propto r^{-2}$ or so (e.g., Jewitt \& Luu 1995; Trujillo et al. 2001), while the KBOs in other models are essentially equidistant (Bernstein et al. 2004). However, Figure 8 shows that a realistic Kuiper Belt would have been eroded from the inside out by the giant planets' gravitational perturbations, which suggests that the earlier models might not apply. However, it turns out that an estimate of the total KBO population does not depend strongly on a particular model's radial variation. As $\S 11$ shows, all three models yield population estimates that are within a factor of $\sim 2$ of each other. This is because the observable KBOs really do inhabit a relatively narrow Belt centered on $r \simeq 45 \mathrm{AU}$, with a radial half-width that is only $\Delta r \sim 4$ AU (see Fig. 8), so the assumption of equidistant KBOs (e.g., Bernstein et al. 2004) appears to be good enough.

It should also be noted that the magnitude interval over which a model Kuiper Belt can be compared to the observed Belt is given by the brightness of those KBOs having reliable orbits, and this sample is currently dominated by bodies having a relatively limited magnitude range of only $21 \lesssim m_{R} \lesssim 24$. Further testing of this model, as well as the development of alternative models of the Belt, would be greatly facilitated if they could be compared to a larger sample of multiopposition KBOs having reliable orbits and also exhibiting a broader range of apparent magnitudes and sizes. This larger KBO sample would be very useful in many ways. For example, it could be used to test the possibility that the various Kuiper Belt subpopulations do exhibit variations in their size distributions (e.g., $\S 6$ ). This larger sample might also permit a better understanding of certain rare and unusual KBOs, such as those that inhabit the Extended Scattered Disk ( $\S 10)$. A deeper understanding of the Kuiper Belt, and what the Belt tells us about the early evolution of the outer solar system, would be facilitated by deeper KBO surveys over larger portions of the sky in a systematic way, which would lead to efficient $\mathrm{KBO}$ recoveries and reliable orbit determinations.

\subsection{Summary of Findings}

1. Accretion models have shown that Kuiper Belt objects must have formed in a dynamically cold environment where the initial KBO seeds had nearly circular and coplanar orbits with 
eccentricities and inclinations $\lesssim 0.001$ (Stern 1996; Kenyon \& Luu 1999). Simulations of Neptune's outward migration into a dynamically cold Kuiper Belt, described in $\S 3$, show that the survivors in the Main Belt still maintain low eccentricities and inclinations. However, this conflicts with the Main Belt's observed $e$ and $\sin i$ values of $\sim 0.1$. This discrepancy suggests that some other process has also stirred up the Kuiper Belt. This stirring event could have occurred prior to or after the onset of planet migration.

2. The existence of several KBOs librating at Neptune's 5:2 resonance suggests that this stirring event occurred prior to the onset of planet migration. Simulations by Chiang et al. (2003) have shown that if Neptune migrates into a stirred-up Kuiper Belt having eccentricities of $e \sim 0.1$, then trapping at Neptune's higher order resonances, such as 5:2, becomes more efficient. This result is confirmed by the higher resolution study of this phenomena described in $\S 4$, which reveals that additional trapping also occurs at a number of exotic mean-motion resonances such as $11: 6,13: 7,13: 6,9: 4,7: 3,12: 5,8: 3,3: 1,7: 2$, and 4:1; such resonances are not populated when Neptune migrates into a dynamically cold disk. Not surprisingly, Neptune's migration into a previously stirred-up Kuiper Belt also accounts for the eccentricities of $e \sim 0.1$ observed in the Main Belt.

3. However, the planet-migration scenario investigated here does not account for the observed KBOs with inclinations above $i \sim 15^{\circ}(\S 5)$, which is the main deficiency of this model. This is a serious discrepancy, since half of all KBOs have inclinations $i>15^{\circ}$ according to the debiased inclination distribution reported by Brown (2001).

4. Neptune's migration into a stirred-up Kuiper Belt traps particles in eccentric orbits at a number of resonances beyond $a=50 \mathrm{AU}$, the most prominent of these being the $5: 2$ and $3: 1$ resonances. Many of these distant particles that are trapped at semimajor axes $a>50$ AU also have perihelia 30 AU $\lesssim q \lesssim$ 40 AU, which is the domain conventionally known as the Scattered Disk. However, $\S 4$ shows that only about $10 \%$ of the simulated particles that inhabit the Scattered Disk or the Extended Scattered Disk (such as the gray zone in Fig. 4, where 50 AU < $a<80 \mathrm{AU}$ and $e>0.25$ ) are truly scattered particles. The vast majority of these particles never had a close encounter with Neptune; rather, they were placed in these wide, eccentric orbits by Neptune's sweeping mean-motion resonances. Note that the origin of these bodies being due to resonant trapping is very distinct from the scattering scenario originally suggested by Duncan \& Levison (1997).

5. Of the $10^{4}$ particles simulated here, only 1 managed to persist over the age of the solar system in the Extended Scattered Disk, which is loosely defined as scattered orbits having perihelia $q \gtrsim 40$ AU. This particle's orbit is qualitatively similar to that of $2000 \mathrm{CR}_{105}$, which has a perihelion of $q=44 \mathrm{AU}$. However, our simulations did not produce any extreme members of the Extended Scattered Disk that were similar to 2003 $\mathrm{VB}_{12}$ (Sedna), which has a perihelion of $q=76 \mathrm{AU}$.

6 . The output of the $N$-body model is coupled to a Monte Carlo model that assigns radii $R$ to the simulated particles according to a power-law cumulative size distribution that varies as $N(R) \propto R^{-Q}$. Magnitudes are computed for the simulation's particles, which then allows us to directly compare the simulated Belt to the observed Belt in a manner that accounts for telescopic selection effects. Section 6 compares the observed abundance of 2:1 objects to known Main Belt objects, and it is shown that the observed 2:1 population is underdense by a factor of 20 relative to model predictions. Similarly, the observed $3: 2$ population is also depleted relative to model expectations.
Another curious feature of the $3: 2$ population is its lower than expected abundance (relative to the Main Belt KBOs) of fainter KBOs having magnitudes $m_{R} \gtrsim 23.5$. Section 6 shows that this dearth of fainter KBOs at the $3: 2$ resonance can be interpreted as a dearth of small bodies, which implies that the $3: 2$ population has a $Q \simeq 2.7$ size distribution that is substantially shallower than the canonical $Q=4.4$ power law that holds for the larger members of the Main Belt.

7. The simulated Centaurs are quite sparse owing to their short dynamical lifetimes; only seven Centaurs were detected in the simulation during its final 2 Gyr. Interestingly, all seven originated at or near Neptune's mean-motion resonances in the Kuiper Belt $(\S 8)$. The model puts an upper limit of $N_{\mathrm{C}} \lesssim 80$ Centaurs having radii larger than $R=50 \mathrm{~km}$, assuming they have a material density $\rho=1 \mathrm{~g} \mathrm{~cm}^{-3}$ and an albedo $p=0.04$. It should be noted that all the simulated Centaurs inhabit heliocentric distances of $r>22 \mathrm{AU}$, while the three Centaurs reported in Sheppard et al. (2000) were detected at $r<19$ AU. If the simulated Centaurs are representative of reality, then this discrepancy in their heliocentric distances may indicate that Centaurs can break up and spawn additional Centaurs (e.g., Pittich \& Rickman 1994) after evolving inward from the Kuiper Belt.

8. This model also estimates that there are at most $N_{\mathrm{T}} \sim$ 1100 Trojans larger than $R=50 \mathrm{~km}$ having a total mass of $M_{\mathrm{T}} \sim$ $5 \times 10^{-4} M_{\oplus}$ orbiting at Neptune's triangular Lagrange points, assuming the usual $\rho=1 \mathrm{~g} \mathrm{~cm}^{-3}$ and $p=0.04$.

9. The absence of any distant KBOs having low eccentricities at $a>50$ AU places tight upper limits on the abundances of any KBOs that might inhabit a hypothetical Outer Belt. Several possible upper limits are inferred from this null result: (1) the primordial density of Outer Belt objects beyond $50 \mathrm{AU}$ may be smaller than the primordial Main Belt density by a factor $f \gtrsim 100$, (2) these distant KBOs may be fainter than $m^{*} \simeq$ 24.5 and thus have radii smaller than $R \simeq 80(p / 0.04)^{-1 / 2} \mathrm{~km}$, (3) the cumulative size distribution of Outer Belt objects may be steep, having a power-law index of $Q>6.0$, or (4) some combination of the above.

10. The luminosity function of the $N$-body/Monte Carlo model is fitted to the KBOs' observed luminosity function, which then yields an estimate of the Belt's total population of $N \sim$ $1.7 \times 10^{5} \mathrm{KBOs}$ larger than $R \simeq 50(p / 0.04)^{-1 / 2} \mathrm{~km}$ with a total mass of $M_{\text {tot }} \sim 0.08\left(\rho / 1 \mathrm{~g} \mathrm{~cm}^{-3}\right)(p / 0.04)^{-3 / 2} M_{\oplus}$. The population and mass of the Belt's various subclasses (e.g., Centaurs, Neptune Trojans, 3:2 and 2:1 populations, the Main Belt, and the Scattered Disk) are also assessed in $\S 11$ and listed in Table 1.

The authors thank Matt Holman for his comments on this paper, Rodney Gomes for discussions of the Neptune-evader mechanism, and Hal Levison for his review of this paper. The derivation of Brouwer's $B$-integral appearing in Appendix A was suggested to us several years ago by Bill Ward. Support for this research was provided to J. M. H. by a Discovery Grant from the Natural Sciences and Engineering Research Council of Canada (NSEAC). Several of the simulations reported here were executed on the Institute for Computational Astrophysics (ICA) Pluto computer cluster, which is funded by a grant from the Canada Foundation for Innovation (CFI). Additional simulations were also performed on the McKenzie computer cluster at the Canadian Institute for Theoretical Astrophysics (CITA) at the University of Toronto; those machines are funded by the CFI, as well as the Ontario Innovation Trust (OIT). An early generation of these simulations was also performed while J. M. H. was 
in residence at the Lunar and Planetary Institute (LPI), and that portion of this research was supported by the National Aeronautics and Space Administration (NASA) via Origins of Solar Systems grant NAG5-10946. R. M. acknowledges research support from NASA's programs in Planetary Geology and Geophysics and in Origins of Solar Systems.

\section{APPENDIX A}

Consider the orbital evolution of a particle trapped by a migrating planet's $j+k: j$ mean-motion resonance. The planet's disturbing function contains the resonant term $R_{\text {res }}=R_{\text {res }}(\alpha$, $\left.e, \phi_{j k}\right)$, where $\alpha$ is the planet/particle semimajor axis ratio for a particle having an eccentricity $e$ and a resonance angle with the form

$$
\phi_{j k}=(j+k) \lambda-j \lambda_{p}-k \tilde{\omega}
$$

where $\lambda$ and $\lambda_{p}$ are the particle and planet's mean longitude and $\tilde{\omega}$ is the particle's longitude of periapse (Murray \& Dermott 1999). The relevant Lagrange planetary equations are

$$
\begin{gathered}
\frac{d a}{d t}=\frac{2}{n a} \frac{\partial R}{\partial \epsilon}, \\
\frac{d e}{d t}=-\frac{\sqrt{1-e^{2}}}{n a^{2} e}\left[\left(1-\sqrt{1-e^{2}}\right) \frac{\partial R}{\partial \epsilon}+\frac{\partial R}{\partial \tilde{\omega}}\right],
\end{gathered}
$$

where $\epsilon=\lambda-n t$ is the particle's mean longitude at epoch, $n$ is the particle's mean motion, $t$ is the time, and coplanar orbits are assumed. According to the averaging principle, the nonresonant terms in the planet's disturbing function are of high frequency and average out during the particle's libration period, and the planet's disturbing function is simply $R=R_{\text {res }}$. The derivatives of $R$ are

$$
\frac{\partial R}{\partial \epsilon}=(j+k) \frac{\partial R_{\mathrm{res}}}{\partial \phi_{j k}}, \quad \frac{\partial R}{\partial \tilde{\omega}}=-k \frac{\partial R_{\mathrm{res}}}{\partial \phi_{j k}} .
$$

With these, the planetary equations (A2) can be combined into a single differential equation,

$$
\frac{d e}{d t}=-\frac{\sqrt{1-e^{2}}}{2 e}\left(\frac{j}{j+k}-\sqrt{1-e^{2}}\right) \frac{1}{a} \frac{d a}{d t} .
$$

It is convenient to replace $e$ with the variable $E=\left(1-e^{2}\right)^{1 / 2}$; since $e d e=-E d E$, the above equation can then be recast as

$$
\frac{d a}{a}=\frac{2 d E}{\gamma-E}
$$

where $\gamma=j /(j+k)$. This differential equation is now easily integrated and yields $\ln a=-2 \ln (\gamma-E)+\ln B$, where the integration constant $B$ can also be expressed as

$$
B=a\left(\sqrt{1-e^{2}}-\frac{j}{j+k}\right)^{2}
$$

(e.g., Hahn \& Ward 1995). The earliest derivation of this integral of the motion known to us is given in Brouwer (1963).
Brouwer originally used this integral to consider the motion of a particle being perturbed by a planet in a static orbit. However, Yu \& Tremaine (1999) recognized that this integral is preserved even when the planet is migrating, which means that equation (A6) can be used to predict the particle's eccentricity as its orbit is expanded by the planet's resonance:

$$
e^{2}(a)=1-\left(\frac{j}{j+k}+\sqrt{\frac{B}{a}}\right)^{2} .
$$

If, prior to capture, the particle is in a circular orbit with an initial semimajor axis $a_{0}$, then $B=a_{0}[k /(j+k)]^{2}$,

$$
e^{2}(a)=1-\left(\frac{j+k \sqrt{a_{0} / a}}{j+k}\right)^{2}
$$

and the particle's eccentricity grows as the particle's orbit expands. An approximate form of this expression is also derived in Malhotra (1993b). Thus, if Neptune's orbital expansion is indeed responsible for the KBOs seen at Neptune's 3:2 resonance at $a=39.4 \mathrm{AU}$ with eccentricities as high as $e \simeq 0.32$ (see Fig. 2), equation (A8) indicates they must first have been orbiting at $a_{0}=28.0 \mathrm{AU}$ at the time of capture and that Neptune was initially orbiting at $a_{\mathrm{N}}=a_{0} \gamma^{2 / 3}=21.4 \mathrm{AU}$, and hence migrated a distance $\Delta_{\mathrm{N}}=8.7 \mathrm{AU}$.

\section{APPENDIX B}

The following shows that the ratio of the observed abundance of any two dynamical classes of KBOs is approximately equal to the ratio of the absolute abundances of the much larger unseen populations. Begin by letting $N_{x}^{\text {obs }}(m)$ be the number of observed KBOs that inhabit some dynamical class $x$ that are brighter than apparent magnitude $m$, where $x$ might represent, for instance, the $3: 2$ population. Also assume that all KBOs have the same heliocentric distance $r$, which is a common assumption (e.g., Irwin et al. 1995; Bernstein et al. 2004) that simplifies this analysis considerably and is actually not a bad assumption, since Figure 8 shows that most of the observed multiopposition KBOs considered here do indeed inhabit a rather narrow belt, with more than half found at heliocentric distances within 3 AU of $r=42$ AU. We also assume that the Kuiper Belt has azimuthal symmetry, which is justified further in Appendix C. We also assume that all $\mathrm{KBO}$ astronomers are observing this Belt largely along the ecliptic, which also is not a bad assumption, since $72 \%$ of the multiopposition KBOs studied here have latitudes of $|\beta|<3^{\circ}$, which is the typical inclination of the Belt's low- $i$ component (Brown 2001). This indicates that the astronomers who discovered most of the KBOs in this sample (Fig. 3, red circles) were observing largely along the Belt's midplane. So, although these assumptions are not rigorously correct in detail, they are good enough to allow us to assess qualitatively the relative abundances of the various KBO populations.

Let $\Sigma_{x}(m)$ represent the sky-plane number density of class $x$ KBOs (i.e., the cumulative luminosity function of class $x$ KBOs), while $\Sigma_{x}^{\prime}(m) \equiv d \Sigma_{x} / d m$ is the differential luminosity function for class $x$. Then the number of observed KBOs brighter than magnitude $m$ is (Bernstein et al. 2004)

$$
N_{x}^{\mathrm{obs}}(m)=\int \Sigma_{x}^{\prime}(m) \Omega(m) \eta_{x}(m) d m
$$


where $\Omega(m)$ is the total solid angle that has been surveyed by all $\mathrm{KBO}$ astronomers to a limiting magnitude $m$, and $\eta_{x}(m)$ is the efficiency at which a KBO of class $x$ and magnitude $m$ is detected. Thus, the number of observed class $x$ KBOs with magnitudes in the interval $m \pm \Delta m / 2$ is

$$
d N_{x}^{\mathrm{obs}}(m) \equiv \frac{d N_{x}^{\mathrm{obs}}}{d m} \Delta m=\Sigma_{x}^{\prime}(m) \Omega(m) \eta_{x}(m) \Delta m
$$

If we were to compare class $x$ KBOs to, for instance, class $y$ KBOs, then the ratio of their observed abundances would be

$$
r_{x: y}(m)=\frac{d N_{x}^{\mathrm{obs}}(m)}{d N_{y}^{\mathrm{obs}}(m)} \simeq \frac{\Sigma_{x}^{\prime}(m)}{\Sigma_{y}^{\prime}(m)}
$$

where it is assumed that the KBO detection efficiency is insensitive to dynamical class, i.e., $\eta_{x}(m) \simeq \eta_{y}(m)$. Note that this ratio depends only on the KBOs' differential luminosity functions $\Sigma^{\prime}(m)$ and that it is insensitive to their discovery details, such as the total solid angle $\Omega(m)$ that the KBO astronomers have surveyed to depth $m$.

The cumulative luminosity function for class $x$ can be written as $\Sigma_{x}(m)=N_{x}(m) / \Omega_{\text {tot }}$, where $\Omega_{\text {tot }}$ is the Kuiper Belt's total solid angle and $N_{x}(m)$ is the cumulative magnitude distribution for class $x$, i.e., the total number of KBOs in class $x$ that are brighter than magnitude $m$. Thus,

$$
\Sigma_{x}^{\prime}(m)=\frac{1}{\Omega_{\mathrm{tot}}}\left(\frac{d N_{x}}{d m}\right)=\frac{1}{\Omega_{\mathrm{tot}}}\left(\frac{d N_{x}}{d R}\right)\left(\frac{d R}{d m}\right),
$$

since a body's magnitude $m$ is a function of its radius $R$ via equation (6), for which

$$
\frac{d N_{x}}{d R}=-\frac{Q N_{x, \text { tot }}}{R_{\min }}\left(\frac{R}{R_{\min }}\right)^{-(Q+1)}
$$

is the differential size distribution for class $x$, which has a total of $N_{x, \text { tot }}$ bodies with radii in the interval $R_{\min }<R<R_{\max }$ (see eq. [4]). Furthermore, since $d R / d m=-R / 5$ (see eq. [6]), the differential luminosity function can be written

$$
\Sigma_{x}^{\prime}(m(R))=\frac{Q N_{x, \text { tot }}}{5 \Omega_{\mathrm{tot}}}\left(\frac{R}{R_{\min }}\right)^{-Q} .
$$

Thus, if any two KBO classes $x$ and $y$ have the same size distribution $Q$, then the ratio of their observed abundances is simply

$$
r_{x: y}(m) \simeq \frac{\Sigma_{x}^{\prime}(m)}{\Sigma_{y}^{\prime}(m)} \simeq \frac{N_{x, \text { tot }}}{N_{y, \text { tot }}} .
$$

In other words, the ratio of the observed abundance of any two classes of KBOs is approximately equal to the ratio of their intrinsic abundances, provided all bodies have the same size distribution $Q$. But if populations $x$ and $y$ have different size distributions, then $r_{x: y}(m)$ varies with $m$ (see $\left.\S 6\right)$.

\section{APPENDIX C}

The Monte Carlo model of $\S 6$ replicates each $N$-body survivor seen in Figure $310^{4}$ times by randomizing the particles' mean anomalies, which results in a model Kuiper Belt with azimuthal symmetry. Thus, the model implicitly assumes that the Kuiper Belt has this same symmetry. However, one might question this assumption, since it is well known that a planet's resonant perturbations can rearrange the longitudes of a smallbody population. For instance, particles at Neptune's 3:2 resonance tend to approach perihelion at longitudes that are $\sim 90^{\circ}$ away from the planet (Malhotra 1995). This is illustrated in Figure 16 (top left), which shows the ecliptic coordinates of all simulated particles that are in or very near Neptune's 3:2 resonance. This figure shows that Neptune tends to arrange the $3: 2$ bodies preferentially away from the Sun-Neptune line, causing regions that lead/trail Neptune by $\pm 90^{\circ}$ to be more densely populated, termed "sweet spots" in Chiang \& Jordan (2002). Figure 16 shows that resonant shepherding also occurs at Neptune's 2:1 resonance, again similar to that seen in Chiang \& Jordan (2002). Red circles indicate the positions of the multiopposition KBOs considered here, which shows that KBOs in or near the $3: 2$ resonance tend to be discovered at longitudes that are roughly $\pm 90^{\circ}$ away from Neptune, as expected.

It is thus possible that Neptune's rearrangement of the Belt might skew our estimate of the resonant populations. If, for example, KBO astronomers were preferentially surveying the Belt along longitudes that are $\pm 90^{\circ}$ away from Neptune, they would detect a rather high sky-plane number density of KBOs in the 3:2 resonance. If we then assumed that this 3:2 number density were uniform about the entire ecliptic, we would overestimate the total $3: 2$ population. Similarly, if astronomers systematically observed the Belt toward Neptune or $180^{\circ}$ away, we would underestimate the $3: 2$ population's average sky-plane density and undercount its total population. But if astronomers surveyed all longitudes with equal frequency, then these competing effects - due to Neptune pushing KBOs away from certain longitudes and toward other longitudes - should wash out, resulting in an estimate of the $3: 2$ population that is approximately reliable.

Figure 16 (top right) shows the ecliptic coordinates of the observed multiopposition Main Belt KBOs. Since the Main Belt population is likely azimuthally symmetric, these red circles should be a good indicator of where astronomers are looking for KBOs. It is quite clear from this figure that these astronomers' lines of sight are not distributed uniformly about the ecliptic. For instance, these KBO hunters tend to avoid the Galactic plane, which passes through the ecliptic along the dashed line. The Main Belt figure also shows that astronomers have avoided a narrow portion of the $3: 2$ population's sweet spot that leads Neptune by about $90^{\circ}$. But it is also quite clear that these same astronomers are not systematically staring at the 3:2 resonance's sweet spots, nor are they systematically avoiding them. Consequently, our simple visual inspection of this KBO sample indicates that our estimate of the $3: 2$ population is not significantly biased toward under- or overcounting the $3: 2$ population, and that the $3: 2$ abundance reported in $\S 11$ is indeed representative. A similar conclusion is also drawn for the much sparser 2:1 population.

Of course, this treatment succeeds only if the Monte Carlo method of $\S 6$ does not alter the particles' radial distribution as they have their mean anomalies randomized by the replication process. However, it is straightforward to show that this is indeed the case. For instance, randomizing the mean anomalies of the $3: 2$ population seen in Figure 16 does not change their radial distribution in any significant way. 


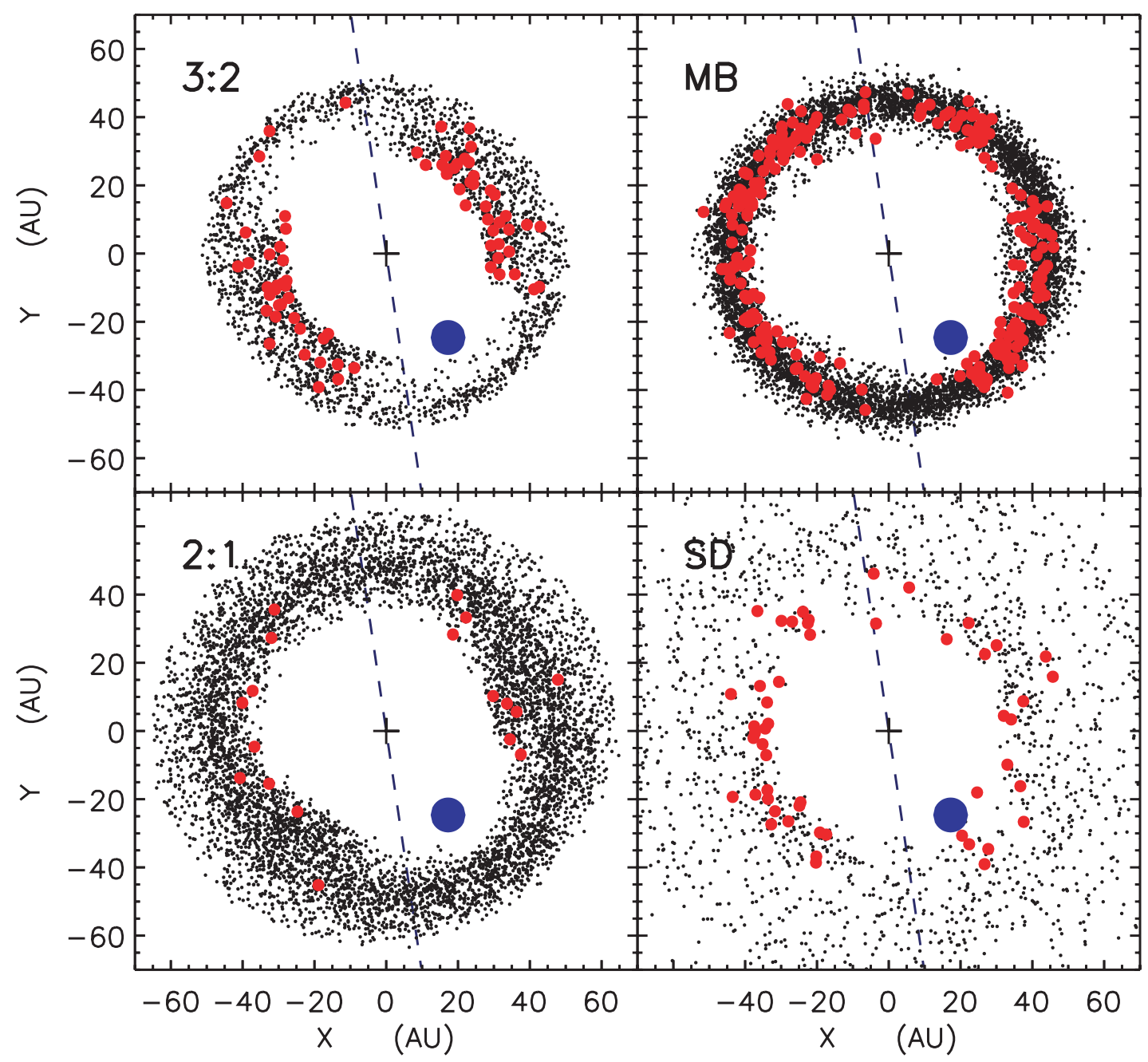

FIG. 16.-Positions of all simulated particles relative to Neptune recorded at all times later than $t=3 \mathrm{Gyr}$ (black dots). Red circles indicate the ecliptic coordinates of the observed multiopposition KBOs, shown for 2000 July 1, which is the date by which half of the sample considered here had been discovered. The blue circle is Neptune's position on this date, and the dashed line shows where the Galactic plane penetrates the ecliptic $x-y$ plane. The particles and KBOs are sorted by their dynamical membership: bodies in or very near the 3:2 resonance (top left), Main Belt (top right), 2:1 resonance (bottom left), and the Scattered Disk objects (bottom right) with perihelia $30 \mathrm{AU}<q<40 \mathrm{AU}$ and $a>48.4 \mathrm{AU}$.

Allen, R. L., Bernstein, G. M., \& Malhotra, R. 2001, ApJ, 549, L241 Altenhoff, W. J., Bertoldi, F., \& Menten, K. M. 2004, A\&A, 415, 771 Bernstein, G. M., Trilling, D. E., Allen, R. L., Brown, M. E., Holman, M., \& Malhotra, R. 2004, AJ, 128, 1364

Borderies, N., \& Goldreich, P. 1984, Celest. Mech., 32, 127

Brouwer, D. 1963, AJ, 68, 152

Brown, M. E. 2001, AJ, 121, 2804

Brown, M. E., Trujillo, C., \& Rabinowitz, D. 2004, ApJ, 617, 645

Chambers, J. E. 1999, MNRAS, 304, 793

Chiang, E. I., \& Jordan, A. B. 2002, AJ, 124, 3430

Chiang, E. I., \& Lithwick, Y. 2005, ApJ, 628, 520

Chiang, E. I., et al. 2003, AJ, 126, 430

Dermott, S. F., Malhotra, R., \& Murray, C. D. 1988, Icarus, 76, 295

Dohnanyi, J. W. 1969, J. Geophys. Res., 74, 2531

Duncan, M., Quinn, T., \& Tremaine, S. 1988, ApJ, 328, L69

Duncan, M. J., \& Levison, H. F. 1997, Science, 276, 1670

Duncan, M. J., Levison, H. F., \& Budd, S. M. 1995, AJ, 110, 3073

Elliot, J. L., et al. 2005, AJ, 129, 1117

Fernández, J. A., \& Brunini, A. 2000, Icarus, 145, 580

Fernández, J. A., \& Ip, W.-H. 1984, Icarus, 58, 109

Franklin, F. A., Lewis, N. K., Soper, P. R., \& Holman, M. J. 2004, AJ, 128, 1391

Gladman, B., Holman, M., Grav, T., Kavelaars, J., Nicholson, P., Aksnes, K., \& Petit, J.-M. 2002, Icarus, 157, 269

Gladman, B., Kavelaars, J. J., Nicholson, P. D., Loredo, T. J., \& Burns, J. A. 1998, AJ, 116, 2042

\section{REFERENCES}

Gomes, R. S. 1997, AJ, 114, 2166 2003, Icarus, 161, 404

Gomes, R. S., Morbidelli, A., \& Levison, H. F. 2004, Icarus, 170, 492 Grundy, W. M., Noll, K. S., \& Stephens, D. C. 2005, Icarus, 176, 184

Hahn, J. M., \& Malhotra, R. 1999, AJ, 117, 3041

Hahn, J. M., \& Ward, W. R. 1995, Lunar Planet. Sci. Conf., 26, 541 2002, Lunar Planet. Sci. Conf., 33, 1930

Holman, M. J., \& Wisdom, J. 1993, AJ, 105, 1987

Ida, S., Larwood, J., \& Burkert, A. 2000, ApJ, 528, 351

Irwin, M., Tremaine, S., \& Zytkow, A. N. 1995, AJ, 110, 3082

Ivezić, Ž., et al. 2001, AJ, 122, 2749

Jewitt, D. C., \& Luu, J. X. 1995, AJ, 109, 1867

Kenyon, S. J., \& Luu, J. X. 1999, AJ, 118, 1101

Kortenkamp, S. J., Malhotra, R., \& Michtchenko, T. 2004, Icarus, 167, 347

Levison, H. F., \& Duncan, M. J. 1997, Icarus, 127, 13 2000, AJ, 120, 2117

Levison, H. F., \& Morbidelli, A. 2003, Nature, 426, 419

Levison, H. F., \& Stern, S. A. 2001, AJ, 121, 1730

Liou, J. C., \& Malhotra, R. 1997, Science, 275, 375

Malhotra, R. 1993a, Icarus, 106, 264

. 1993b, Nature, 365, 819

1995, AJ, 110, 420

1996, AJ, 111, 504

Malhotra, R., \& Williams, J. G. 1997, in Pluto and Charon, ed. S. A. Stern \& D. J.

Tholen (Tucson: Univ. Arizona Press), 127

Malyshkin, L., \& Tremaine, S. 1999, Icarus, 141, 341 
Morbidelli, A., Jacob, C., \& Petit, J.-M. 2002, Icarus, 157, 241

Morbidelli, A., \& Levison, H. F. 2004, AJ, 128, 2564

Murray, C. D., \& Dermott, S. F. 1999, Solar System Dynamics (Princeton: Princeton Univ. Press)

Nagasawa, M., \& Ida, S. 2000, AJ, 120, 3311

Nesvorný, D., \& Dones, L. 2002, Icarus, 160, 271

Pittich, E. M., \& Rickman, H. 1994, A\&A, 281, 579

Rauch, K. P., \& Holman, M. 1999, AJ, 117, 1087

Sheppard, S. S., Jewitt, D. C., Trujillo, C. A., Brown, M. J. I., \& Ashley, M. C. B. 2000, AJ, 120, 2687

Sheppard, S. S., Trujillo, C., Jewitt, D. C., \& Marsden, B. G. 2005, Minor Planet Electron. Circ., 2005-H21

Stern, A., \& Campins, H. 1996, Nature, 382, 507

Stern, S. A. 1996, AJ, 112, 1203
Stuart, J. S., \& Binzel, R. P. 2004, Icarus, 170, 295

Tanga, P., Cellino, A., Michel, P., Zappalà, V., Paolicchi, P., \& dell'Oro, A. 1999, Icarus, 141, 65

Thommes, E. W., Duncan, M. J., \& Levison, H. F. 1999, Nature, 402, 635 2002, AJ, 123, 2862

Tiscareno, M. S., \& Malhotra, R. 2003, AJ, 126, 3122

Trujillo, C. A., \& Brown, M. E. 2001, ApJ, 554, L95

Trujillo, C. A., Jewitt, D. C., \& Luu, J. X. 2001, AJ, 122, 457

Tsiganis, K., Gomes, R., Morbidelli, A., \& Levison, H. F. 2005, Nature, 435, 459

Ward, W. R. 1981, Icarus, 47, 234

Yu, Q., \& Tremaine, S. 1999, AJ, 118, 1873

Zhou, L., Sun, Y., Zhou, J., Zheng, J., \& Valtonen, M. 2002, MNRAS, 336, 520 